\title{
Mesenchymal Stem Cells Ameliorate Interstitial Fibrosis in Adenine-induced Nephropathy Based on Renal Proteomics
}

Huajun Tang

Department of Human Anatomy, School of Basic Medical Sciences, Southwest Medical University

\section{Peiyue Zhang}

Department of Human Anatomy, School of Basic Medical Sciences, Southwest Medical University

\section{Lianlin Zeng}

Department of Human Anatomy, School of Basic Medical Sciences, Southwest Medical University

\section{Yu Zhao}

Department of Human Anatomy, School of Basic Medical Sciences, Southwest Medical University Libo Xie ( $\nabla$ piterxielibo@163.com )

Department of Urology, Sichuan Clinical Research Center for Nephropathy, The Affiliated Hospital of Southwest Medical University

\section{Bo Chen ( $\nabla$ cb0402022@swmu.edu.cn )}

Department of Human Anatomy, School of Basic Medical Sciences, Southwest Medical University https://orcid.org/0000-0003-1810-1849

\section{Research Article}

Keywords: Adenine, Mesenchymal stem cells, Interstitial fibrosis, Proteomics

Posted Date: October 30th, 2020

DOI: https://doi.org/10.21203/rs.3.rs-100450/v1

License: (c) (1) This work is licensed under a Creative Commons Attribution 4.0 International License. Read Full License 


\section{Abstract}

Background: Tubulointerstitial fibrosis (TIF) is one of the main pathological features of various progressive renal damages and chronic kidney diseases. Mesenchymal stromal cells (MSCs) have been verified with significant improvement in the therapy of fibrosis diseases, but the mechanism is still unclear. We attempted to explore the new mechanism and therapeutic target of MSCs against renal fibrosis based on renal proteomics.

Methods: TIF model was induced by adenine gavage. Bone marrow derived MSCs was injected by tail vein after modeling. Fibrosis biomarkers or extracellular matrix proteins and histopathological change were assessed by Masson staining, Sirius red staining, immunohistochemistry, and western blot. Renal proteomics was analyzed using iTRAQ-based mass spectrometry.

Results: MSCs treatment clearly decreased the expression of a-SMA, collagen type I, II, III, TGF- $\beta 1$, pSmad2/3, IL-6, IL-1 $\beta$, and TNFa compared with model rats, while p38 MAPK increased. 6,213 proteins were identified, but only 40 proteins exhibited significant differences (30 upregulated, 10 downregulated) compared MSCs group with the model group. Bioinformatics analysis revealed that these proteins play important roles in the proliferation, inflammatory and immune responses, apoptosis, phagosome, etc. According to literatures and bioinformatics analysis, the most markedly downregulated protein, galectin3, was further assessed by quantitative PCR and western blot in renal tissues. Galectin3 levels were downregulated in adenine-induced renal tissues and TGF- $\beta 1$ induced tubular epithelial cells and interstitial fibroblasts in consistent with iTRAQ after MSCs treatment.

Conclusion: The founds suggest that galectin3 maybe involves in the antifibrotic mechanisms of MSCs therapy for tubulointerstitial fibrosis as well as a possible therapeutic target.

\section{Introduction}

Adenine-induced nephropathy is a rat model of human tubulointerstitial fibrosis (TIF), which is the main pathological feature of various progressive renal damage and the final common pathway for chronic kidney disease (CKD). TIF is characterized by infiltration of renal interstitial monocytes and lymphocytes, myofibroblast activation, excessive deposition of extracellular matrix (ECM), and sclerosis ${ }^{[1]}$. Adenine is mainly used to participate in the synthesis of DNA and RNA and has been widely used for the treatment of patients with leukopenia ${ }^{[2]}$. However, it causes severe nephrotoxicity and fibrosis because of the high accumulation in the kidney. Adenine produces 2, 8-dihydroxyadenine through the action of xanthine oxidase, which deposits in the glomeruli and the interstitium, forming granulomatous inflammation and blocking the renal tubules leading to cystic dilatation of renal tubules and kidney failure ${ }^{[3]}$. Adenineinduced CKD rats exhibited anemia, uremia, decreased renal function, increased infiltration of inflammatory cells, tubular atrophy, and fibrosis ${ }^{[1]}$. 
Mesenchymal stem cell-based therapy has emerged as a promising way for antifibrosis in the latest 10 years ${ }^{[4]}$. MSCs secrete a wide range of soluble cytokines that are helpful for anti-fibrosis, anti-apoptosis, immunomodulation, and tissue repair ${ }^{[5]}$. Several studies have shown that MSCs ameliorate renal fibrosis in adenine, cisplatin, adriamycin-induced animal models and an ischemia-reperfusion injury model ${ }^{[6-9]}$. However, it has not been fully elucidated that the mechanisms of MSCs for improving renal fibrosis and function. In this study, we examined how MSCs ameliorate renal fibrosis in adenine-induced rats based on isobaric tags for relative quantification proteomics (iTRAQ) and further verification in vivo and in vitro.

\section{Materials And Methods}

\subsection{Isolation, culture, and identification of MSCs}

Rat bone marrow-derived MSCs were obtained from the femur and tibia of healthy male Sprague Dawley rats (100-120 g). These cells were cultured in low glucose DMEM containing 10\% FBS (Gibco, Invitrogen, New York, USA), $2.5 \mathrm{mM}$ L-glutamine, and penicillin/streptomycin (Gibco, Invitrogen, New York, USA) in a $5 \% \mathrm{CO}_{2}$ incubator at $37{ }^{\circ} \mathrm{C}$. When the cultures reached $90 \%$ confluence, MSCs were harvested using $0.25 \%$ trypsin-EDTA (Thermo Fisher Scientific, Waltham, MA, USA). MSCs were subcultured at the ratio of 1:3. For all experiments, MSCs (passages 3-4) were collected and washed with sterile saline to achieve a single cell suspension for model treatment or analysis. To identify MSCs, the cells were incubated with the following antibodies against surface antigens (Abcam,UK): CD90-FITC (ab226), CD29-PE/Cy7 (ab95622), CD45-FITC (ab33916) and CD34-FITC (11-0341-82, eBioscience, USA). Finally, MSCs were washed and resuspended in $0.4 \mathrm{~mL}$ of PBS for analysis using a BD FACSCalibur flow cytometer and Cell Quest software (BD Biosciences, San Jose, CA, USA). Rat MSCs induction and differentiation were carried out using Oil red $O$ staining to verify adipogenesis, using Alizarin red staining to confirm osteogenesis.

\subsection{Adenine-induced nephropathy and administration of MSCs}

Male Sprague Dawley rats (250-280 g, n=40) were purchased from Dashuo Biotechnology Co. LTD (Chengdu, China). Rats were housed in specific pathogen-free conditions at $24^{\circ} \mathrm{C}$ with $50 \%$ relative humidity under a $12 \mathrm{~h}$ light/dark cycle. All rats were randomly divided into three groups according to the random number table: Control group (Control), Adenine-induced nephropathy group (Adenine), and MSCs treatment group (Adenine+MSCs) ( $\mathrm{n}=10$ in Control group, $\mathrm{n}=15$ in Adenine group and Adenine+MSCs group). Adenine group was induced by intragastric administration of $150 \mathrm{mg} / \mathrm{kg}$ adenine (Sigma Aldrich, USA) for 20 days. In the meanwhile, adenine gavage was suspended for 2 days at day 10 and continued. Adenine+MSCs group was injected with $1 \mathrm{~mL}$ of MSCs suspension $\left(2.0 \times 10^{6} \mathrm{MSCs} / \mathrm{kg}\right)$ via the tail vein on day 3 after adenine gavage ends. Control group was instead injected with the same volume of saline. After MSCs treatment for 5 days, proteinuria level was measured with a kit from Thermo Fisher Scientific, and serum creatinine and urea nitrogen levels were measured with a kit from AmyJet Scientific Inc (Wuhan, Hubei, China). Serum creatinine, urea nitrogen, and proteinuria levels were measured by a Beckman Analyzer II (Beckman Instruments, Inc. USA). All animal studies were approved by the 
Southwest Medical University Animal Experimentation Ethics Committee and were performed in accordance with the approved guidelines.

\subsection{Histological analysis and immunohistochemistry staining}

The kidneys were fixed in phosphate buffer solution containing $4 \%$ paraformaldehyde and then embedded in paraffin. Tissue sections $(5 \mu \mathrm{m})$ were stained with Hematoxylin -Eosin staining, Masson trichrome (Solarbio, Beijing, China) and Sirius Red staining (Yeasen Biotechnology Co. LTD., Shanghai, China) according to the instructions and viewed through a microscope. Tubular injury was assessed by the grading of tubular necrosis, cast formation, and tubular dilation. Scoring or positive area was quantified in 6-8 equivalent cortical HPFs (200x) from each rat by Image Pro Plus Software 6.0 (Media Cybernetics, Silver Spring, USA) and calculated as follows: 0, none; 1, $\leq 10 \% ; 2,11 \%-25 \% ; 3,26 \%-45 \%$; , $46 \%-75 \%$; and $5,>76 \%$ according to the literature ${ }^{[10]}$.

For immunohistochemistry staining, kidney sections were blocked with $3 \% \mathrm{H}_{2} \mathrm{O}_{2}$ in PBS for 15 min and nonspecific sites were blocked with $5 \%$ goat serum albumin for $30 \mathrm{~min}$ at room temperature. Then, slices were incubated overnight at $4{ }^{\circ} \mathrm{C}$ with 1:100 diluted rabbit anti-a-SMA antibody (ab5694, Abcam, UK), 1: 50 diluted rabbit collagen I (ab90395, Abcam, UK), 1:200 diluted rabbit Collagen II antibody (ab34712, Abcam, UK), 1:100 diluted rabbit Collagen III antibody (ab34710, Abcam, UK), and and 1:400 diluted rabbit p38 MAPK antibody (\#8690, Cell Signaling, USA), respectively. After that, the HRP conjugated goat anti-rabbit IgG secondary antibody (ab6721, Abcam, UK) was added and incubated for 1.5 hours at room temperature. After washing with PBS and counterstaining with 3, 3'-diamnobenzidine (DAB), then the slices were placed in xylene to penetrate, concentration gradient alcohol to dehydrate, and images were examined by a light microscope. Positive staining was quantified in 6 equivalent cortical HPFs (200x) by Image Pro Plus 6.0 software. Protein semiquantitative analysis was presented as integrated optical density (IOD sum).

\subsection{The Cytokine Antibody Array}

The serum specimens were processed according to the guidelines specified by the Raybiotech Antibody Microarray protocol (GSR-CYT-3-1; Raybiotech, Atlanta, Georgia State, USA). All chemical reagents and solvents were obtained from the Raybiotech Antibody Microarray kit and Wayne Biotechnologies Inc., $\triangle$ Shanghai, China $\mathbb{X}$. The protein concentration of each specimen was determined using a BCA protein assay kit (Beyotime, Shanghai, China). A rat cytokine array kit was used to compare cytokine expression in serum between control group, adenine group and adenine+MSCs group, $n=5$ in each group.

Standardized quantities of each protein specimen were loaded into identical cytokine antibody subarrays. Following incubation at room temperature for 2 hours, the subarrays were washed with the included wash buffer according to the operating instructions. The detection process was then performed using a biotin antibody cocktail as well as Cy3-Streptavidin. The subarrays were scanned on a GenePix 4000B microarray scanner and the raw data were readed by GenePix Pro 6.0 software (Axon Instruments, USA). A total of 27 cytokines were detected on the GSR-CYT-3-1 chip, and each antibody on the chip was set up 
four technical repetitions. During the data analysis, the mean value of four replicates was first calculated as the signal value of each factor, then the signal value was normalized to a positive control to allow comparison between subarrays, and finally the concentration was quantified by using the normalized data. The intergroup ratio of 27 factors was calculated, and the $P$ values between groups were analyzed by $t$-TEST (double-tailed).

\subsection{Renal tissue proteomics}

\subsubsection{Protein isolation, digestion, labeling with iTRAQ reagents}

Rats were sacrificed at day 5 after MSCs intervention to carry out renal proteomic analysis based on isobaric tags for relative and absolute quantification (iTRAQ). The workflow of the study is presented in Figure 1. Forty-five kidney tissues were washed twice with ice-cold PBS and then homogenized with MP homogenizer (MP Fastprep-24, 5G) in SDT pyrolysis solution (4\% SDS, 1 mM DTT, $150 \mathrm{mM}$ Tris-HCl pH 8.0, and protease inhibitor). After ultrasonication, the homogenate was incubated for $15 \mathrm{~min}$ in boiling water. The crude extract was then centrifugated at $14,000 \times \mathrm{g}$ at $25^{\circ} \mathrm{C}$ for $15 \mathrm{~min}$, the protein concentration was measured by a BCA protein assay kit. The samples were stored at $-80^{\circ} \mathrm{C}$.

Protein digestion was performed according to the filter-aided sample preparation procedure described by Wisniewski ${ }^{[11]}$, and the resulting peptide mixture was labeled using the 8-plex iTRAQ reagent according to the manufacturer's instructions (Applied Biosystems, Foster City, CA). Briefly, $30 \mu \mathrm{L}$ of protein digestion for each sample were added into DTT to the final concentration of $100 \mathrm{mM}$, boiling water for $5 \mathrm{~min}$, cooling to room temperature. The detergent, DTT, and other low-molecular-weight components were removed using $200 \mu \mathrm{L}$ UA Buffer ( $8 \mathrm{M}$ Urea and $150 \mathrm{mM}$ Tris-HCl, $\mathrm{pH}$ 8.0) by repeated ultrafiltration. Next, $100 \mu \mathrm{L}$ IAA buffer ( $0.1 \mathrm{M}$ iodoacetamide in UA Buffer) was added to block to reduce cysteine residues and the samples were incubated for $30 \mathrm{~min}$ in darkness. The filters were washed with $100 \mu \mathrm{L}$ UA buffer two times, washed twice with $100 \mu \mathrm{L}$ dissolution buffer. Finally, the protein suspensions were digested with $4 \mu \mathrm{g}$ trypsin (Promega, Madison, WI) in $40 \mu \mathrm{L}$ dissolution overnight at $37^{\circ} \mathrm{C}$ and the resulting peptides were collected as a filtrate. The peptide content was estimated by UV light spectral density at $280 \mathrm{~nm}$ with Nano Drop 2000. $100 \mu \mathrm{g}$ peptides from each sample were labeled according to the iTRAQ Reagent-8 plex Multiplex Kit (AB Sciex, UK). The peptides were, respectively, mixed as a pool. The peptides from all groups were, respectively, mixed as a pool and then equally divided into three fractions (Control group: A1 and A2; Adenine group: B1, B2 and B3; Adenine+MSCs group: C1, C2 and C3). A standard pool comprising a mixture of equal amounts of protein derived from all samples served as an internal control. The samples were labeled as A1-113, A2-114, B1-115, B2-116, B3-117, C1-118, C2-119, and C3-121, and were multiplexed and vacuum dried. For labeling, each iTRAQ reagent was dissolved in $70 \mu \mathrm{L}$ of ethanol and added to the respective peptide mixture. This experiment was done with three biological replicates.

\subsubsection{Mass spectrometry analysis}


All the labeled samples were mixed with equal amount. Next, the labeled samples were fractionated using a Agilent 1260 infinity II high-performance liquid chromatography (HPLC) system (Thermo Dinoex Ultimate 3000 BioRS) equipped with a XBridge Peptide BEH C18 (130Å, $5 \mu \mathrm{m}, 4.6 \mathrm{~mm} \times 100 \mathrm{~mm})$ column. LC-MS/MS analysis was performed with an Q Exactive Plus LC-MS/MS system (Thermo Scientific, Waltham, Massachusetts, USA).

\subsubsection{Bioinformatic analysis}

The mass spectrometry data was analyzed using Proteome Discoverer 2.1 (Thermo Scientific) and Mascot 2.5 sortware, peptide identifications were made using the Paragon algorithm searching against the uniprot Rattus Norvegicus protein database (http://www.uniprot.org/). Only the peptide FDR value which set to less than 0.01 was contained in ITRAQ labeling quantification, and proteins with an average fold change larger than 1.2 times were considered significantly differentially expressed for further analysis. To determine the biological and functional properties of the identified proteins, the identified protein sequences were mapped with Gene Ontology Terms (http://geneontology.org/). In brief, a homology search was firstly performed for all identified sequences with a localized NCBI BLAST+ (ncbiblast-2.2.28+-win32.exe) program against NCBI database. The e-value was set to less than $1 \mathrm{e}-3$, and the best hit for each query sequence was accounted for $\mathrm{GO}$ term matching. The informations on molecular function, cellular components, and biological process were acquired by searching with terms and gene products. The $\mathrm{GO}$ term matching was performed with Blast2go Command Line. Pathway analysis specifying the relationships between the interacting molecules was made by keyword search in the KEGG GENES database (http://www.kegg.jp/) and KAAS (KEGG Automatic Annotation Server Ver. 2.1) online tool (http://www.genome.jp/tools/kaas/). The pathway enrichment statistics were performed by Fisher's exact test, and those with a corrected $p$ value $<0.05$ were regarded as the most significant pathways.

\subsection{Western Blot}

About $100 \mathrm{mg}$ of kidney tissue was lysated in ice-cold RIPA lysis buffer (Beyotime, China) containing 1 mM PMSF. The concentration of protein was determined by a BCA assay kit. About $50 \mu \mathrm{g}$ protein samples were loaded in 10\% SDS-PAGE gels and transferred onto the PVDF membrane (Millipore, USA). After blocking with $5 \%$ skim milk, the membrane was incubated overnight at $4{ }^{\circ} \mathrm{C}$ with the following antibodies: TGF $\beta 1$ (1:2000, Proteintech, USA), GAPDH (1:50000, Proteintech, USA), a-SMA (1:5000, Proteintech, USA), Galectin3 (1:1000, Cell Signaling, USA), p38 MAPK (1:1000, Abcam, UK), phosphoSamd2/3 (1:1000, Cell Signaling, USA), Smad2/3 (1:1000, Cell Signaling, USA), IL-6 (1:1000, Proteintech, USA), IL-1 $\beta$ (1:1000, Bioworld, China), and TNFa (1:1000, Proteintech, USA) antibodies. The bolts were incubated with horseradish peroxidase (HRP)-conjugated goat-anti rabbit or mouse secondary antibodies for $1 \mathrm{~h}$, and the membranes reacted with chemiluminescence HRP substrate (Solarbio, China) and exposed to the ChemiScope 6000 Exp image system (CliNX, Shanghai, China) for visualization of protein bands. The protein bands were quantified using the NIH ImageJ Software.

\subsection{Fluorescence quantitative polymerase chain reaction}


For further verification, we selected the most clearly downregulated protein (galectin3) compared adenine group with adenine+MSCs group according to bioinformatics analysis and references ${ }^{[12]}$. Total RNA were extracted using trizol (Invitrogen, USA) from renal tissues in all groups. First-strand cDNA syntheses were performed from total RNA by reverse transcription using the Eastep RT Master Mix Kit (Promega, Shanghai, China). Fluorescence quantitative PCR amplifications were performed at $95^{\circ} \mathrm{C}$ for $10 \mathrm{sec}, 60^{\circ} \mathrm{C}$ for $15 \mathrm{sec}$ using rat Galectin3 using the Eastep qPCR Master Mix kit (Promega, Shanghai, China). GAPDH was used as an internal control. The design of the oligonucleotide primer sequences based on: Galectin3, sense 5'-aacgacatcgccttccac-3', and antisense 5'-cccagttattgtcctgcttc-3'; GAPDH, sense 5'-

gcaagttcaacggcacag-3' and antisense 5'-gccagtagactccacgacat-3'. Fluorescence quantitative PCR was performed on LightCycle480 (Roche, Basel, Switzerland) in triplicate. Specificity of the PCR products was confirmed by analysis of the dissociation curve. In addition, the amplicons' expected size was confirmed by analysis of the PCR products on $1 \%$ agarose gels, subsequently visualized under UV light. The relative mRNA levels were calculated using the $2^{-\triangle \Delta C t}$ method after normalization with GAPDH as a housekeeping gene. All data for RNA expression analysis were calculated using the $2^{-\Delta \Delta C t}$ method.

\subsection{NRK-49F and NRK-52E Cells culture and treatment}

Rat renal fibroblast cells (NRK-49F) and rat renal tubular epithelial cells (NRK-52) were purchased from the Beina Chuanglian Biotechnology Research Institute (BNBIO, Beijing, China) and separately maintained in MEM and DMEM medium (Gibco/Life Technologies, Grand Island, NY) supplemented with $10 \%$ FBS (Gibco/Life Technologies). Cells that reached approximately $50 \%$ confluence were used for in vitro experiments.

To test the effect of MSCs on galectin3 protein expression induced by TGF- $\beta 1$ in two cells. We first prepared MSCs conditioned medium (MSCs-CM). MSCs (Passages 3, 80\% confluence) were incubated with serum-free DMEM low-glucose for $24 \mathrm{~h}$ at $37^{\circ} \mathrm{C}$ before MSCs-CM supernatant collection.

Supernatants were then centrifuged at $2,000 \mathrm{rpm}$ at $4^{\circ} \mathrm{C}$ for $5 \mathrm{~min}$ and the cell debris were removed with a $0.22 \mu \mathrm{m}$ disposable filter. NRK-49F and NRK-52E were, respectively, seeded into $6 \mathrm{~cm}$ sterile dishes and randomly divided into five groups: (1) Normal group; (2) TGF- $\beta 1$-induced group; (3) TGF- $\beta 1+T D 139$ (a specific galectin3 inhibitor, MCE, NJ, USA) group; (4) TGF- $\beta 1+50 \%$ MSC-CM group; (5) TGF$\beta 1+$ TD139+50\% MSC-CM group. TGF- $\beta 1$-induced group was serum starved for $12 \mathrm{~h}$, followed by incubation with recombinant human TGF- $\beta 1$ ( $20 \mathrm{ng} / \mathrm{mL} \otimes$ PeproTech, Rocky Hill, NJ, USA) for $48 \mathrm{~h}$. TD139 was pretreated for $2 \mathrm{~h}$ before TGF- $\beta 1$ treatment. $50 \%$ MSCs-CM was added to the dishes for $48 \mathrm{~h}$. The cell lysates were used for western blot analysis.

\subsection{Statistical analysis}

Data are presented as mean $\pm S D$ of at least three biological repeation. Normal distribution of data was checked by means of the Shapiro-Wilk test and a Levene statistic test was performed to check the homogeneity of variances. To determine statistical significance, statistical analysis was performed using 
one-way ANOVA (GraphPad Software, San Diego, CA, USA), followed by the Bonferroni post hoc testing to analyze differences between groups. $P<0.05$ was considered significant.

\section{Results}

\subsection{MSCs features}

Bone marrow-derived MSCs showed a typical spindle-shaped appearance (Fig. 2A), and the ability to differentiate into osteocytes and adipocytes (Fig. 2B, 2C). Flow cytometric analysis revealed MSCs showed high expression levels of CD29 and CD90 and low expression levels of CD34 and CD45 (Fig. 2D). These findings confirmed the presence of a MSC phenotype.

\subsection{MSCs ameliorated renal function in Adenine-induced nephropathy}

We examined the therapeutic effects of MSCs on renal injury in adenine-treated rats. Adenine was carried out intragastric administration continuously for 20 days and MSCs were transplanted on day 3 , and creatinine, urea nitrogen, and $24 \mathrm{~h}$ urinary protein were analyzed on days 0,20 , and 30 . Kidney index (Kidney weight/Body weight $\times 100 \%$ ) of adenine-treated rats increased, exhibited typically large white kidneys (Fig. 3E), and MSCs prevented these increases (Fig. 3A). Adenine increased the levels of serum urea nitrogen (Fig. 3B), creatinine (Fig. 3C), and $24 \mathrm{~h}$ urinary proteins (Fig. 3D). These effects were prevented by MSCs.

\subsection{MSC treatment ameliorated renal fibrosis in Adenine-induced rats.}

Adenine leaded to cystic dilatation of renal tubules, inflammatory cell infiltration, and renal damage scores increase, in contrast, the pathologic changes in MSC-treated rats were milder (Fig. 4A, D). The Masson's trichrome staining (Fig. 4B, E) and Sirus red staining (Fig. 4C, F) of renal tissues showed that renal interstitial extracellular matrix increased in the Adenine-induced group, while the renal fibrosis was improved by MSC treatment in Adenine+MSC group. The immunohistochemical staining revealed that Collagen I, II, III was majorly expressed in the tubulointerstitium in the Adenine group, while the expression of Collagen I, II, III was greatly reduced in Adenine+MSC group (Fig. 5A-C, E-G).

To further explore the role of MSCs against renal fibrosis, we examined the typical molecules involved in TGF- $\beta$ /Smad signaling pathway. The immunohistochemistry staining showed that the expressions of $a-$ SMA in the kidneys of adenine-induced rats were clearly increased and then decreased after MSCs administration (Fig. 5D, H). Western blot also showed that adenine significantly increased the expression of a-SMA, TGF- $\beta 1$, and phosphorylated Smad2/3, and MSCs markedly inhibited this enhancement (Fig. 6). These data suggest that adenine impaired renal tubules and induced renal interstitial fibrosis, which can be ameliorated by MSCs.

\subsection{MSCs activated p38 MAPK signaling and reduced inflammation in Adenine-induced rats}


The immunohistochemical staining and western blot showed adenine decreased p38 MAPK expression compared with the control group, and MSCs treatment surely enhanced p38 MAPK expression compared with the Adenine group (Fig. 7A). The expressions of IL-6, IL-1 3 , and TNF- $a$ in kidney tissues were all significantly elevated in the adenine group, whereas these cytokine levels were indeed reduced by MSC treatment (Fig. 7B). Microarray analysis of cytokine antibodies revealed that a total of 17 cytokines increased in the serum, such as CINC-1, CINC-2, CINC-3, GM-CSF, ICAM-1, IFNy, IL-1a, IL-2, IL-6, IL-10, LSelectin, MCP-1, PDGF-AA, Prolactin R, RAGE, TCK-1, TIMP-1, and VEGF, and a total of 8 cytokines decreased, including $\beta-N G F, C N T F$, Fractalkine, IL-1 $\beta$, IL-4, IL-13, LIX, and TNFa in adenine group. Among them, there were significantly statistical differences both increases in ICAM-1, IL-10, and L-Selectin and reduction in IL-1 $\beta$. While MSCs treatment reduced the serum levels of 18 upregulated cytokines and increased the serum levels of 8 downregulated cytokines, but there was no statistical difference. In addition, adenine increased the serum level of B7-2 and decreased the serum level of TCK-1, but MSCs treatment further increased B7-2 level and decreased TCK-1 level without statistical differences (Fig. 7C).

\subsection{Effect of MSCs on the Adenine-induced rat kidney proteome}

Using untargeted proteomic analysis, a total of 58,016 peptides matching 6,213 proteins ( $\geq$ one or more unique peptides with an FDR less than 1\%) were identified. Using a threshold of 1.2-fold change and $p<0.05$ between groups was considered as differentially expressed protein. A total of 5,873 proteins were identified in the quantitative proteomic study. MSCs treatment finitely affected the profile of the renal proteome, only 40 proteins were found differentially expressed compared with adenine group (Table 1). Using Cluster 3.0 software classified the two dimensions of sample and protein expression simultaneously (distance algorithm: Euclid; connection mode: average linkage). Finally, Java Trewview software was used to generate the volcano plots and hierarchical clustering heatmaps between adenine group and adenine+MSCs group (Figure 8A, B). Compared with the Adenine group, 30 proteins were upregulated and 10 downregulated in the MSCs group (Table 2). The majority of biological process that upregulated proteins involved are biological process, cellular process, metabolic process, cellular metabolic process, organic substance metabolic process, single-organism process, primary metabolic process, single-organism cellular process, macromolecule metabolic process and cellular component organization or biogenesis. Meanwhile, the downregulated proteins involved in response to stimulus, biological process, cellular process, single-organism process, biological regulation, regulation of biological process, single-organism cellular process, cellular response to stimulus, regulation of cellular process, and multicellular organismal process. The $\mathrm{GO}$ term of these differently expressed proteins was classified between adenine group and adenine+MSCs group (Figure 8C). The pathway analysis of all upregulated or down-regulated proteins was also shown in Figures 9A and 9B. The top seven pathways identified were the N-Glycan biosynthesis (Mgat3), MAPK signaling pathway (Nme6), bisphenol degradation (Pon3), aminobenzoate degradation (Pon3), thiamine metabolism (Thtpa), steroid hormone biosynthesis (Cyp21a1), and signaling pathways regulating pluripotency of stem cells (Pcgf2) (Table 3). Among them, enrichment analysis showed that there were significantly statistical differences of bisphenol degradation and aminobenzoate degradation in adenine group and adenine+MSCs group, the $p$ value was 0.02 and 0.04 , respectively (Figures $9 C, 10$ ). 
Table 1 Protein quantitative statistics

\begin{tabular}{cccc}
\hline Comparisons & Up- & Down- & All- \\
\hline Adenine vs. Control & 2027 & 1029 & 3056 \\
Adenine+MSCs vs. Control & 1855 & 922 & 2777 \\
Adenine+MSCs vs. Adenine & 30 & 10 & 40 \\
Total & 3885 & 1961 & 5873 \\
\hline
\end{tabular}

Table 2 Differentially expressed proteins between Adenine+MSCs group and Adenine group 


\begin{tabular}{|c|c|c|c|c|}
\hline Accession & Gene Name & $\begin{array}{l}\text { Adenine+MSCs group } \\
\text { vs. Adenine group ratio }\end{array}$ & Regulatec & IPvalue \\
\hline$\overline{\mathrm{B} 0 \mathrm{BN} 83}$ & Armc1 & 3.52 & $\mathrm{Up}$ & $\leq 0.001$ \\
\hline Q02527 & Mgat3 & 1.54 & Up & 0.027 \\
\hline Q6AXR6 & Gsto1 & 1.35 & $\mathrm{Up}$ & 0.017 \\
\hline Q9EP88 & Slc25a14 & 1.34 & Up & 0.043 \\
\hline A0A0G2JZC6 & Arhgef11 & 1.33 & Up & 0.003 \\
\hline D4AD58 & Etfrf1 & 1.31 & Up & 0.033 \\
\hline Q6P777 & Mvb12a & 1.31 & $\mathrm{Up}$ & 0.021 \\
\hline D3ZPW6 & Lage3 & 1.30 & $\mathrm{Up}$ & 0.011 \\
\hline Q64724 & LOC103689942 & 1.30 & Up & 0.026 \\
\hline D3ZMW3 & Msto1 & 1.30 & Up & 0.014 \\
\hline B5DEJ9 & Sbf2 & 1.30 & $\mathrm{Up}$ & 0.006 \\
\hline A0A0G2JSK1 & Serpina3c & 1.29 & Up & 0.003 \\
\hline Q4QQU5 & Yipf6 & 1.28 & Up & 0.020 \\
\hline D4A386 & Cldn2 & 1.27 & Up & 0.042 \\
\hline P31211 & Serpina 6 & 1.26 & Up & 0.013 \\
\hline Q68FP2 & Pon3 & 1.26 & Up & 0.017 \\
\hline Q53B90 & Rab43 & 1.25 & Up & 0.031 \\
\hline D3ZV40 & Unk & 1.23 & Up & 0.005 \\
\hline D4AB26 & Smc6 & 1.23 & Up & 0.005 \\
\hline P11530 & Dmd & 1.23 & $\mathrm{Up}$ & 0.037 \\
\hline A0A0G2K3M6 & Atp9b & 1.22 & Up & 0.017 \\
\hline D3ZGN7 & Mical3 & 1.22 & Up & 0.011 \\
\hline D3ZVK1 & Mcm8 & 1.22 & Up & 0.005 \\
\hline F1LRG0 & Сур21a1 & 1.22 & Up & 0.001 \\
\hline A0A0G2KB52 & Map7 & 1.21 & $\mathrm{Up}$ & 0.002 \\
\hline B2RZ82 & Pcgf2 & 1.21 & Up & 0.043 \\
\hline R9PXW5 & Nme6 & 1.20 & Up & 0.035 \\
\hline A0A0G2JTF6 & Thtpa & 1.20 & Up & 0.040 \\
\hline D3ZIT7 & LOC103689975 & 1.20 & $\mathrm{Up}$ & 0.026 \\
\hline P47967 & Lgals5 & 1.20 & Up & 0.048 \\
\hline Q1WIM1 & Cadm4 & 0.83 & Down & 0.002 \\
\hline D3ZZR3 & Ctss & 0.79 & Down & 0.016 \\
\hline D3ZH40 & Otud7b & 0.79 & Down & 0.020 \\
\hline Q9JK15 & Adap2 & 0.79 & Down & 0.033 \\
\hline G3V6E7 & Fmod & 0.77 & Down & 0.024 \\
\hline A1L1L9 & Tmem65 & 0.76 & Down & 0.030 \\
\hline P27590 & Umod & 0.73 & Down & 0.030 \\
\hline D4A416 & Clptm1l & 0.67 & Down & 0.010 \\
\hline Q6NYB8 & Ifi47 & 0.59 & Down & 0.009 \\
\hline A0A0G2JXN6 & Galectin3 & 0.58 & Down & 0.020 \\
\hline
\end{tabular}

Table 3. List of main KEGG pathways between Adenine group and Ade+MSCs group 


\begin{tabular}{llll}
\hline N-Glycan biosynthesis & 1 & Q02527 (Mgat3) & 0.14 \\
MAPK signaling pathway & 1 & R9PXW5 (Nme6) & 0.07 \\
Bisphenol degradation & 1 & Q68FP2 (Pon3) & 0.02 \\
Aminobenzoate degradation & 1 & Q68FP2 (Pon3) & 0.04 \\
Thiamine metabolism & 1 & A0A0G2JTF6 (Thtpa) & 0.06 \\
Steroid hormone biosynthesis & 1 & F1LRG0 (Cyp21a1) \\
Signaling pathways regulating pluripotency of & & B2RZ82 (Pcgf2) & 0.12 \\
stem cells & 1 & 0.17 \\
\hline
\end{tabular}

\subsection{MSCs-CM protected NRK-52E and NRK-49F against TGF- $\beta 1$ induced fibrosis by reducing galectin3 expression}

To verify whether MSCs which protected NRK-52E and NRK-49F against TGF- $\beta 1$ induced fibrosis have a relationship with galectin3, quantitative PCR and western blot showed that adenine increased mRNA and protein expression of galectin3 compared with adenine group and MSCs treatment markedly decreased these in renal tissues, which were consistent with iTRAQ-based and western blot results $(p<0.05$, Figure 11). In vitro, western blot showed MSCs-CM also downregulated galectin3 expression in TGF- $\beta 1$ induced NRK-52E and NRK-49F. TD139 pretreatment further reduced galectin3 expression.

\section{Discussion}

Mesenchymal stem cell-based therapies have been shown to confer renal protection in several models of acute kidney injury (AKI) and chronic kidney disease (CKD ${ }^{[13,14]}$. Most preclinical and clinical trials have demonstrated the safety and efficacy of MSCs in protecting against renal dysfunction ${ }^{[15-17]}$. MSC therapy is becoming an attractive strategy for renal repair, and the potential of MSCs based therapy or ameliorating CKD or AKI is only beginning to be elucidated. Several recent studies show the capacity of exogenously administered MSCs or MSCs conditioned medium to dramatically reduce tubulointerstitial fibrosis, preserve peritubular capillary density, and prevent epithelial mesenchymal transition in multiple different models of chronic renal injury ${ }^{[19-21]}$. Our results support these evidences, and show a significant reduction in extracellular matrix components, such as collagen type la, collagen type II, and collagen type III; inflammation-related factors, IL-1 $\beta$, IL-6, TFNa, myofibroblast activation marker, a-SMA and increase in proliferation-related signal, p38 MAPK protein expression in adenine-induced nephropathy after MSCs early intervention. To date, the mechanism of MSCs contributed to the renoprotection is involved in paracrine cytokines, growth factors, and immuoregulation ${ }^{[4]}$, but no research has focused on the renal proteomic profile of MSCs therapy for adenine-induced interstitial fibrosis, and may make a valuable contribution towards the comprehension of the molecular mechanisms involved in MSCs which alleviated renal fibrosis.

In this study, iTRAQ combined with Q Exactive Plus LC-MS/MS was used to identify the differentially regulated proteins based on a threshold of 1.2 -fold change and $p<0.05$ between groups. We found that MSCs had a very limited influence on the renal proteome comparing adenine group with adenine+MSCs 
group, only a total of 40 proteins were detected, of which 30 proteins were significantly upregulated and 10 proteins downregulated. According to the reference ${ }^{[22-25]}$ and bioinformatics analysis, galectin3 was one of the significantly downregulated proteins after MSCs treatment. Thus, glaectin3 was further verified by fluorescence quantitative PCR analysis and western blot in renal tissues, and further determined in renal tubular epithelial cells (NRK-52E) and renal interstitial fibroblasts (NEK-49F) induced by human recombinant TGF- $\beta 1$. Our results were in consistent with the ITRAQ results. Besides that, these differentially expressed proteins were involved in a multitude of biological processes including acute inflammatory responses, proliferation, inflammatory response, apoptosis, phagosome, immune response, regulation of the biosynthesis and biological function of glycoprotein oligosaccharides, and so on. Next, we focus on galectin3, MSCs and renal interstitial fibrosis. Therefore, the mechanism of anti-fibrosis of MSCs has not been concerned about galectin3.

Galectin-3 proteins (also known as Lgals3) were localized to the nucleus and mitochondrion, which involved in acute inflammatory responses including neutrophil activation and adhesion, chemoattractant of monocytes and macrophages, opsonization of apoptotic neutrophils, and activation of mast cells (https://www.genecards.org/). Galectin3 protein has recently been deemed as a possible biomarker of inflammation and renal fibrosis and plays a pivotal role in interstitial fibrosis and progression of chronic kidney disease, and it promotes nephrogenesis during development ${ }^{[26]}$. Elevated serum levels of galectin3 have been associated with a higher risk of incident CKD and renal dysfunction ${ }^{[27]}$. Galectin3 can be secreted extracellularly but can also shuttle into the nucleus. Extracellular galectin3 modulates important interactions between epithelial cells and the extracellular matrix and plays a role in the embryonic development of collecting ducts. In contrast, intracellular galectin3 is important for cell survival due to its ability to block the intrinsic apoptotic pathway, while intranuclear galectin3 promotes cell proliferation ${ }^{\text {[28- }}$

${ }^{30]}$. Our experiments proved that MSCs reduced the mRNA and protein expression of galectin3 in adenineinduced interstitial fibrosis kidney. We also determined that galectin3 was downregulated in renal tubular epithelial cells and renal interstitial fibroblasts induced by TGF- $\beta 1$ after MSCs-CM treatment. A specific galectin3 inhibitor, TD139 pretreatment and MSCs further reduced galectin3 expression. However, little is known about the regulatory mechanisms of galectin3 during antifibrosis of mesenchymal stem cells and need to be further explored. Our data provided a new insight and possible therapeutic targets in the antifibrosis of MSCs, which may have a certain valuable in the understanding of the pathogenesis of adenine-induced interstitial fibrosis.

\section{Conclusions}

In summary, we depicted the differentially expressed proteins in the early period of MSCs treatment for adenine-induced nephropathy by ITRAQ-based proteomic analysis. We found MSCs had very limited influence on the renal proteome, only 40 proteins were differentially expressed compared adenine group with MSCs treatment. The antifibrosis effect of MSCs may attribute to the proliferation, immune response, inflammatory response, apoptosis, phagosome, and the like. MSCs, which protect against adenine-induced interstitial fibrosis, have a certain relationship with galectin3 downregulation. Our data 
provided a new insight into MSCs treatment for interstitial fibrosis and may be valuable in furthering our understanding of the pathogenesis of adenine-induced kidney injury and need to be further explored.

\section{Abbreviations}

TIF: Tubulointerstitial fibrosis; MSCs: Mesenchymal stem cells; MSC-CM: MSCs conditioned medium; ITRAQ: isobaric tags for relative and absolute quantitation; CKD: chronic kidney disease; ecm:

extracellular matrix; FBS: Fetal bovine serum; DMEM: Dulbecco's modified eagle medium; MAPK: Mitogenactivated protein kinase; PBS: Phosphate buffer solution; HPFs: High power fields; SDT: 4\% SDS, 1 mM DTT, and 150 mM Tris-HCl; SDS: Sodium dodecyl sulfate; DTT: Dithiothreitol; BCA: Bicinchoninic acid; UA: 8 M Urea and 150 mM Tris-HCl; HPLC: High-performance liquid chromatography; LC-MS/MS: liquid chromatography tandem mass spectrometry; HRP: Horseradish peroxidase; CINC: Cytokine-induced neutrophil chemoattractant; GM-CSF: Granulocyte-macrophage colony-stimulating factor; ICAM-1: Intercellular adhesion molecule-1; INFY: Interferon gamma; MCP-1: Monocyte chemoattractant protein-1; PDGF: Platelet-derived growth factor; RAGE: Advanced glycosylation end-product specific receptor; TIMP1: Tissue inhibitor of matrix metalloproteinase-1; VEGF: Vascular endothelial growth factor; NGF: Nerve growth factor; CNTF: Ciliary neurotrophic factor; LIX: LPS-induced CXC chemokine; TCK-1: Thymus Chemokine-1; B7-2: CD86; TNFa: Tumor necrosis factor a.

\section{Declarations}

\section{Acknowledgements}

We thank Shanghai Genechem Co., LTD for iTRAQ technological assistance and subsequent bioinformatics analysis.

\section{Authors' contributions}

BC contributed to the conception, design, writing and the critical review of the manuscript. HJT and PYZ contributed to the modeling, primary MSCs culture and flow phenotype identification, treatment, and proteomics sample collection. LLZ and YZ were responsible for the immunohistochemical staining, western blot, and PCR. LBX was responsible for collection, assembly of data, and data analysis. All authors read and approved the final manuscript.

\section{Funding}

This work was supported by Science and Technology Department of Sichuan Province (No.2018JY0490), Education Department of Sichuan province (No. 18ZA0524), the Health and Family Planning Commission of Sichuan province (No. 16PJ540), Luzhou Science and Technology Bureau (No. 2016-S-65 (1/9)), National Innovation and Entrepreneurship Program for College Students (No. 201816032012), and Hospital project of traditional Chinese Medicine of Southwest Medical University (No. 2015-20).

\section{Availability of data and materials}


Antibody microarray data and renal proteomics data generated and/or analysed during this study are included in this published article. Data sharing is not applicable to this article as no datasets were generated or analysed during the current study. However, the data that support the findings of this study are available from the corresponding author upon reasonable request.

\section{Ethics approval and consent to participate}

The experiment protocols were approved by the Ethic Committee of Southwest Medical University (reference number SWMU).

\section{Consent for publication}

Not applicable

\section{Conflicts of interest}

We declare that we have no financial and personal relationships with other people or organizations that can inappropriately influence our work. There is no professional or other personal interest in any product, service, and/or company that could be construed as influencing the position presented in the manuscript entitled.

\section{References}

1 E. Vazquez-Mendez, Y. Gutierrez-Mercado, E. Mendieta-Condado, et al. Recombinant Erythropoietin Provides Protection against Renal Fibrosis in Adenine-Induced Chronic Kidney Disease. Mediators Inflamm. 2020;2020(8937657

2 T. Tomita, H. Goto, K. Sumiya, et al. Efficacy of Adenine in the Treatment of Leukopenia and Neutropenia Associated with an Overdose of Antipsychotics or Discontinuation of Lithium Carbonate Administration: Three Case Studies. Clin Psychopharmacol Neurosci. 2016;14(4):391-395

3 A. C. Boon, A. K. Lam, V. Gopalan, et al. Endogenously elevated bilirubin modulates kidney function and protects from circulating oxidative stress in a rat model of adenine-induced kidney failure. Sci Rep. 2015;5(15482

4 E. El Agha, R. Kramann, R. K. Schneider, et al. Mesenchymal Stem Cells in Fibrotic Disease. Cell Stem Cell. 2017;21(2):166-177

5 R. E. B. Fitzsimmons, M. S. Mazurek, A. Soos, et al. Mesenchymal Stromal/Stem Cells in Regenerative Medicine and Tissue Engineering. Stem Cells Int. 2018;2018(8031718

6 S. Yokote, Y. Katsuoka, A. Yamada, et al. Effect of adipose-derived mesenchymal stem cell transplantation on vascular calcification in rats with adenine-induced kidney disease. Sci Rep. 2017;7(1):14036 
7 H. H. Anan, R. A. Zidan, M. A. Shaheen, et al. Therapeutic efficacy of bone marrow derived mesenchymal stromal cells versus losartan on adriamycin-induced renal cortical injury in adult albino rats. Cytotherapy. 2016;18(8):970-984

8 R. Moghadasali, M. Azarnia, M. Hajinasrollah, et al. Intra-renal arterial injection of autologous bone marrow mesenchymal stromal cells ameliorates cisplatin-induced acute kidney injury in a rhesus Macaque mulatta monkey model. Cytotherapy. 2014;16(6):734-749

9 H. Cao, Y. Cheng, H. Gao, et al. In Vivo Tracking of Mesenchymal Stem Cell-Derived Extracellular Vesicles Improving Mitochondrial Function in Renal Ischemia-Reperfusion Injury. ACS Nano. 2020;14(4):4014-4026

10 C. H. Chen, B. C. Cheng, K. H. Chen, et al. Combination therapy of exendin-4 and allogenic adiposederived mesenchymal stem cell preserved renal function in a chronic kidney disease and sepsis syndrome setting in rats. Oncotarget. 2017;8(59):100002-100020

11 Z. Yang, X. Shen, D. Chen, et al. Toward a Universal Sample Preparation Method for Denaturing TopDown Proteomics of Complex Proteomes. J Proteome Res. 2020;19(8):3315-3325

12 R. Dong, M. Zhang, Q. Hu, et al. Galectin-3 as a novel biomarker for disease diagnosis and a target for therapy (Review). Int J Mol Med. 2018;41(2):599-614

13 F. Zhu, O. L. S. Chong Lee Shin, G. Pei, et al. Adipose-derived mesenchymal stem cells employed exosomes to attenuate AKI-CKD transition through tubular epithelial cell dependent Sox9 activation. Oncotarget. 2017;8(41):70707-70726

14 M. Tang, K. Zhang, Y. Li, et al. Mesenchymal stem cells alleviate acute kidney injury by downregulating C5a/C5aR pathway activation. Int Urol Nephrol. 2018;50(8):1545-1553

15 A. Makhlough, S. Shekarchian, R. Moghadasali, et al. Bone marrow-mesenchymal stromal cell infusion in patients with chronic kidney disease: A safety study with 18 months of follow-up. Cytotherapy. 2018;20(5):660-669

16 J. M. Quimby, T. L. Webb, L. M. Habenicht, et al. Safety and efficacy of intravenous infusion of allogeneic cryopreserved mesenchymal stem cells for treatment of chronic kidney disease in cats: results of three sequential pilot studies. Stem Cell Res Ther. 2013;4(2):48

17 T. Squillaro, G. Peluso and U. Galderisi. Clinical Trials With Mesenchymal Stem Cells: An Update. Cell Transplant. 2016;25(5):829-848

18 J. Zheng, Q. Wang, W. Leng, et al. Bone marrowderived mesenchymal stem cellconditioned medium attenuates tubulointerstitial fibrosis by inhibiting monocyte mobilization in an irreversible model of unilateral ureteral obstruction. Mol Med Rep. 2018;17(6):7701-7707 
19 P. Yu, Z. Wang, Y. Liu, et al. Marrow Mesenchymal Stem Cells Effectively Reduce Histologic Changes in a Rat Model of Chronic Renal Allograft Rejection. Transplant Proc. 2017;49(9):2194-2203

20 S. Tanaka, T. Tanaka and M. Nangaku. Hypoxia and Dysregulated Angiogenesis in Kidney Disease. Kidney Dis (Basel). 2015;1(1):80-89

21 B. Liu, F. X. Ding, Y. Liu, et al. Human umbilical cord-derived mesenchymal stem cells conditioned medium attenuate interstitial fibrosis and stimulate the repair of tubular epithelial cells in an irreversible model of unilateral ureteral obstruction. Nephrology (Carlton). 2018;23(8):728-736

22 T. Zhang, S. Cao, H. Yang, et al. Prognostic impact of galectin-3 in chronic kidney disease patients: a systematic review and meta-analysis. Int Urol Nephrol. 2019;51(6):1005-1011

23 M. L. Alam, R. Katz, K. A. Bellovich, et al. Soluble ST2 and Galectin-3 and Progression of CKD. Kidney Int Rep. 2019;4(1):103-111

24 C. M. Rebholz, E. Selvin, M. Liang, et al. Plasma galectin-3 levels are associated with the risk of incident chronic kidney disease. Kidney Int. 2018;93(1):252-259

25 H. Y. Li, S. Yang, J. C. Li, et al. Galectin 3 inhibition attenuates renal injury progression in cisplatininduced nephrotoxicity. Biosci Rep. 2018;38(6):

26 H. Francois and C. Chatziantoniou. Renal fibrosis: Recent translational aspects. Matrix Biol. 2018;6869(318-332

27 E. Martinez-Martinez, J. Ibarrola, L. Calvier, et al. Galectin-3 Blockade Reduces Renal Fibrosis in Two Normotensive Experimental Models of Renal Damage. PLoS One. 2016;11(11):e0166272

28 V. Desmedt, S. Desmedt, J. R. Delanghe, et al. Galectin-3 in Renal Pathology: More Than Just an Innocent Bystander. Am J Nephrol. 2016;43(5):305-317

29 S. C. Chen and P. L. Kuo. The Role of Galectin-3 in the Kidneys. Int J Mol Sci. 2016;17(4):565

30 A. Hara, M. Niwa, K. Noguchi, et al. Galectin-3 as a Next-Generation Biomarker for Detecting Early Stage of Various Diseases. Biomolecules. 2020;10(3):

\section{Figures}




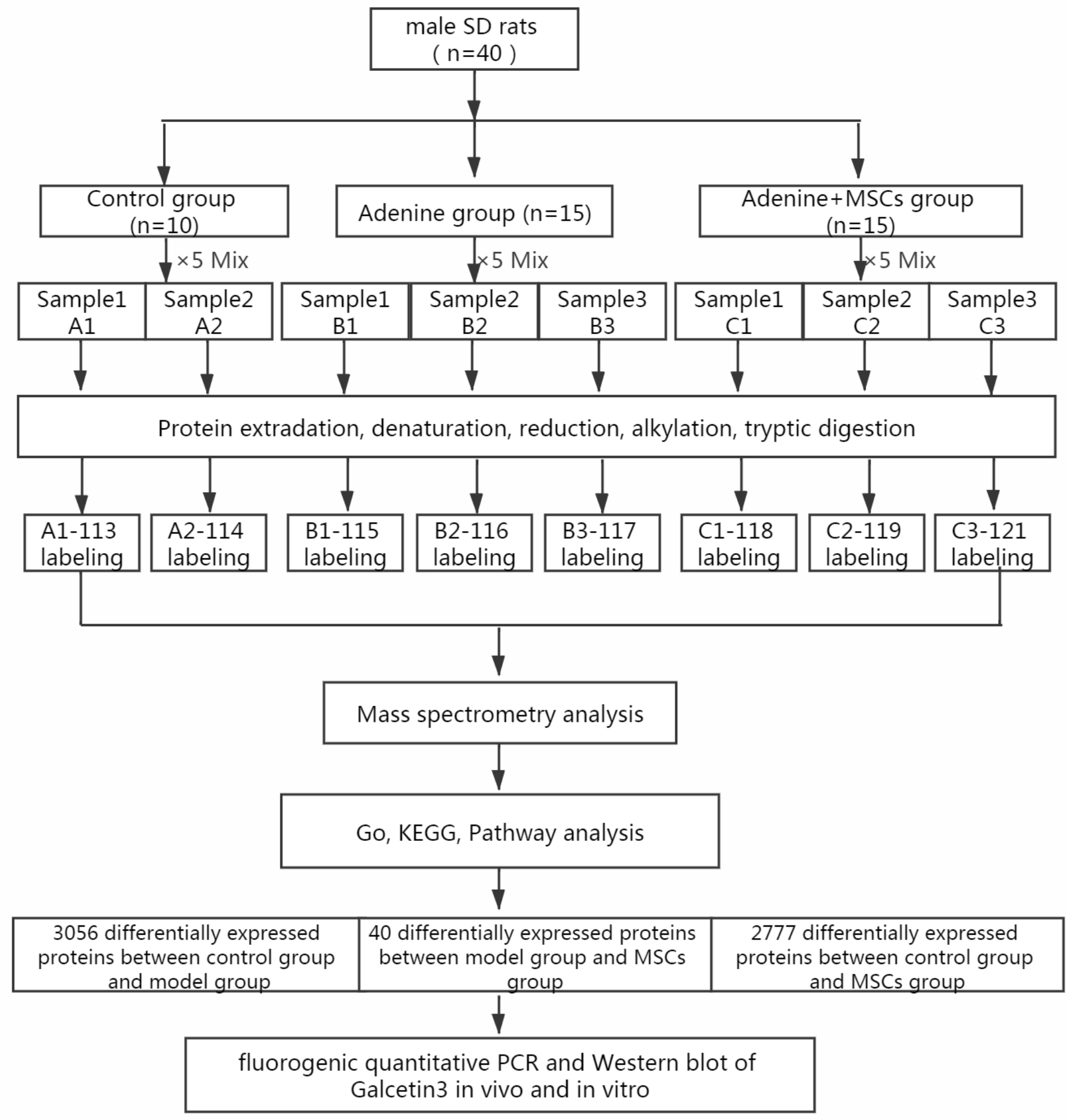

\section{Figure 1}

Diagram of iTRAQ proteomics experimental design. Male Sprague Dawley rats were induced by adenine gavage for 20 days and MSCs treatment for 5 days. Kidney tissues were collected, protein extracted, pooled before iTRAQ labeling and subjected to mass spectrometric analysis. Eight iTRAQ labels were used: 113 and 114 for the $A 1$ and $A 2,115,116$, and 117 for the $B 1, B 2$ and $B 3$, and 118, 119, and 121 for the C1, C2 and C3, respectively. Bioinformatics analysis (GO, KEGG Pathway) was used to examine 
differential expression proteins. Mass spectrometry results were further confirmed by assessing galectin3 protein with western blot and Q-PCR in kidney tissues and in NRK-52E and NRK-49F induced by human recombinant TGF $\beta 1$.
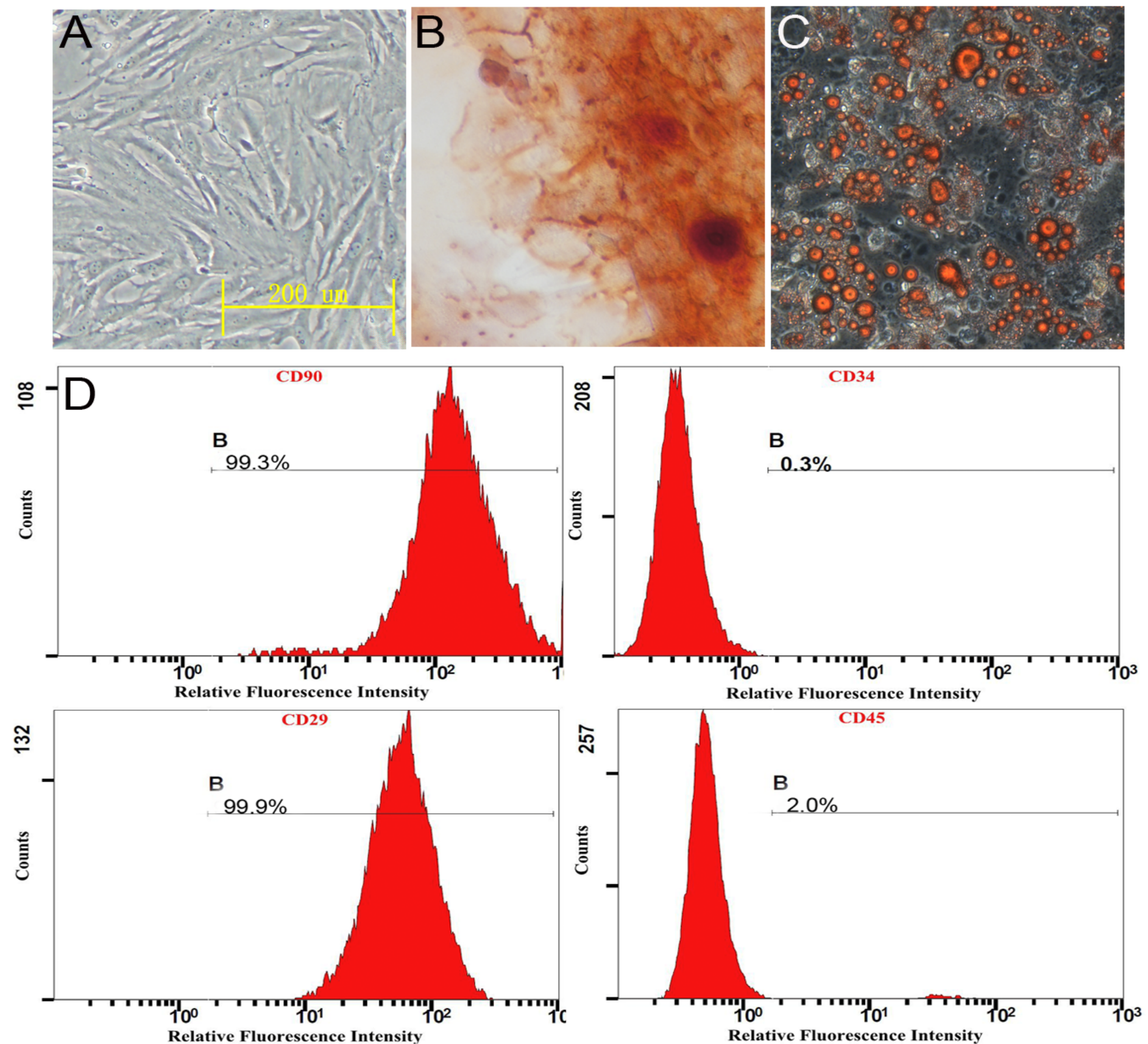

Figure 2

Characteristics of rat bone marrow derived-MSCs. A. MSCs showed a typical spindle-shaped appearance and undifferentiated MSCs. B. MSCs displayed multilineage differentiation potential, osteocytes, as evidenced by the presence of calcium deposits stained with Alizarin Red staining. C. MSCs differentiated into adipocytes, as indicated by the presence of lipid droplets stained with Oil Red O; Scale bar: $200 \mu \mathrm{m} \mathrm{D.}$ 
Immunophenotypic characterization of MSCs (passage 3) was performed by flow cytometry. MSCs associated with markers were positive for CD29 and CD90 and were negative for CD34 and CD45.
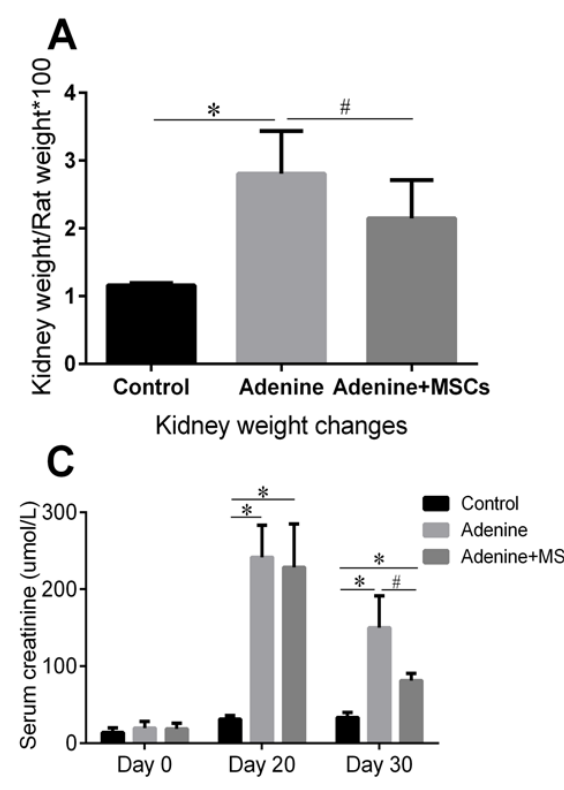

B

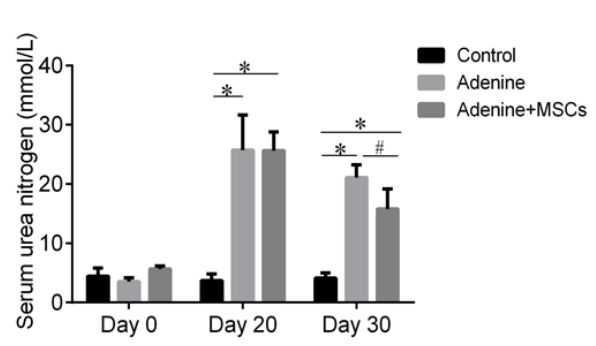

D

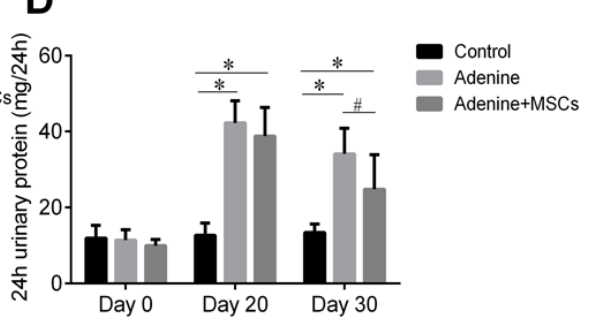

E

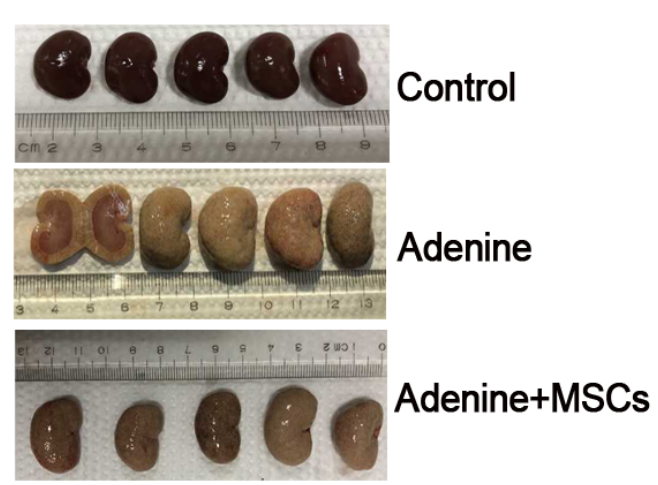

\section{Figure 3}

MSCs treatment improved the renal function indexes of adenine-induced rats. A. Kidney index (kidney weight/bodyweight $\times 100 \%$ ). B. Serum urea nitrogen level. C. Serum creatinine level. D. 24-h urinary protein. E. Holistic view of rat kidney. $\mathrm{N}=5$ (per group), data are presented as mean $\pm \mathrm{SD}$. analyzed by one-way ANOVA followed by Bonferroni post hoc testing. ${ }^{*} \mathrm{P}<0.05$, vs. Control group, $\# \mathrm{P}<0.05$, vs. Adenine group. 


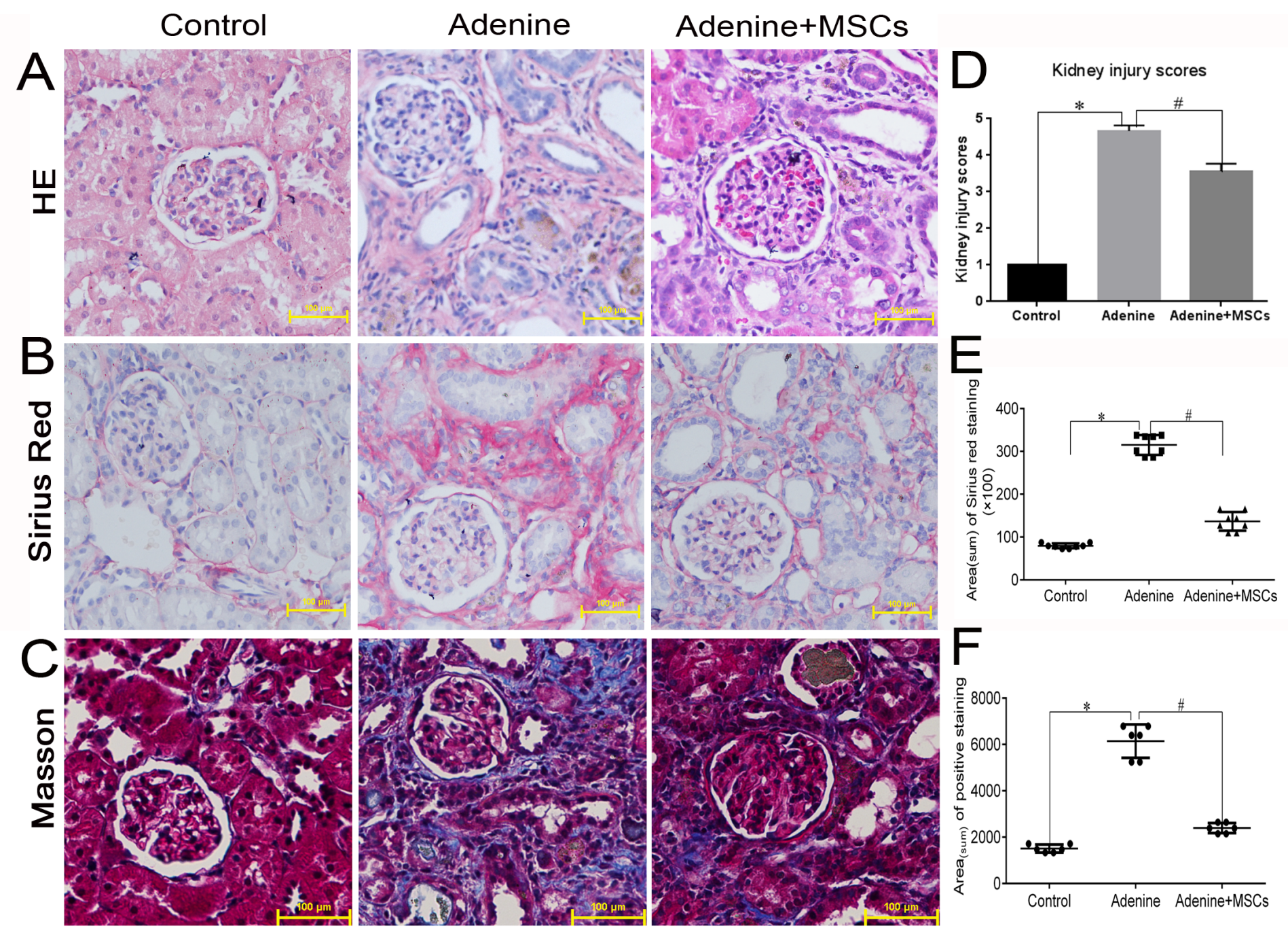

Figure 4

MSCs treatement reduced kidney injury scores and collagen components of extracellular matrix in the tubulointerstitium by histopathology analysis of renal tissues from adenine-induced rats. A. Hematoxylineosin (H\&E) staining. B. Masson staining. C. Sirus Red staining. Representative histochemical staining images of Sirus Red staining and Masson trichrome staining images in kidney sections at 5 days postMSCs treatment (scale bar, $100 \mu \mathrm{m})$. The statistical graph shows the area(sum) of the positive area $(\mathrm{n}=$ 6-8 in each group) by the Image Pro Plus 6.0 software. $N=5$ (per group), data are presented as mean \pm $S D$, analyzed by one-way ANOVA followed by Bonferroni post hoc testing. $* P<0.05$, vs. Control group, $\# \mathrm{P}<0.05$, vs. Adenine group. 

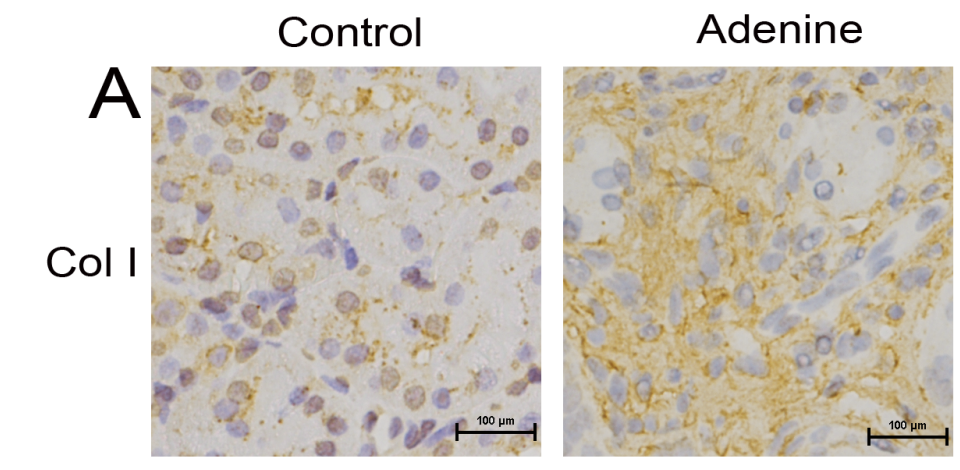

\section{Adenine+MSCs}
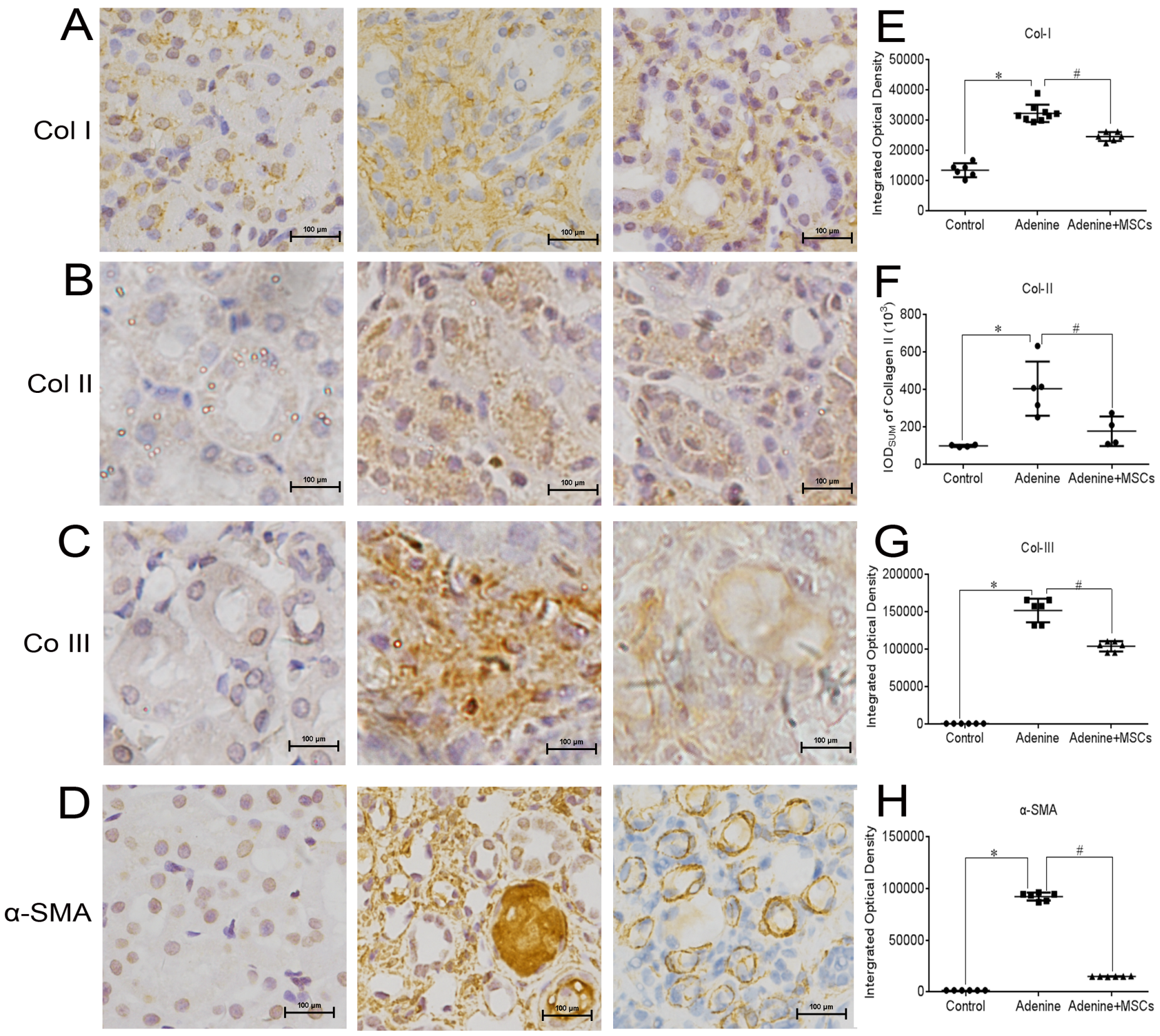

Figure 5

MSCs treatment alleviated the tubulointerstitial fibrosis in adenine-induced rats. Immunohistochemistry of collagen type I (A), collagen type II (B), collagen III type (C) and a-SMA (D) in the tubulointerstitium. Representative immunohistochemical staining images of collagen type I-, II-, III-, and a-SMA positive areas in kidney sections at 5 days post-MSCs treatment (scale bar, $100 \mu \mathrm{m}$ ). The statistical graph shows the integrated optical density (IOD) of collagen type I-, II-, III-, and a-SMA positive areas ( $\mathrm{n}=6-8$ in each group) by the Image Pro Plus 6.0 software. $N=5$ (per group), data are presented as mean $\pm S D$, analyzed by one- 
way ANOVA followed by Bonferroni post hoc testing. ${ }^{\star} \mathrm{P}<0.05$, vs. control group, \#P $<0.05$ vs. adenine group.
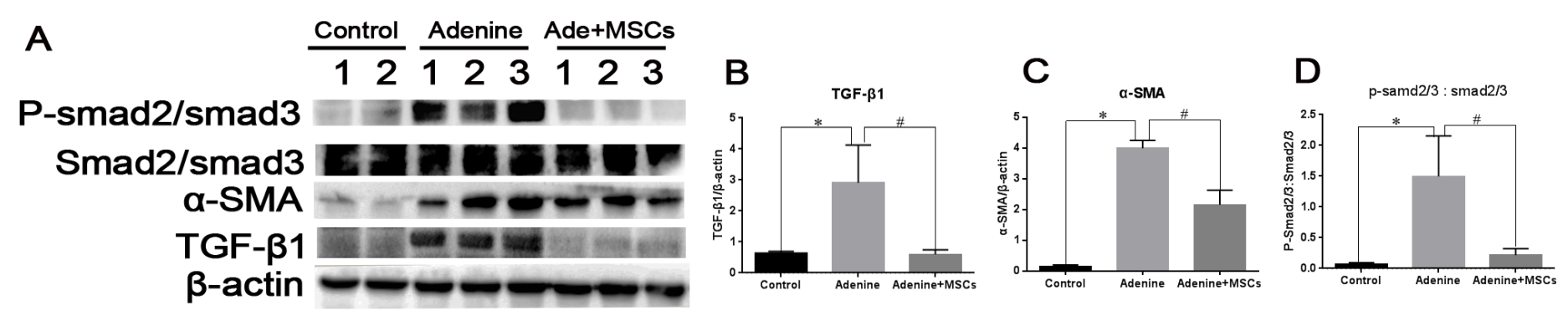

\section{Figure 6}

MSC treatment ameliorated TGF- $\beta 1 /$ Smad signaling pathway in adenine-induced rats by western blot analysis of $\alpha-S M A, P-S m a d 2 / 3, S m a d 2 / 3$, and TGF- $\beta 1$ in kidney tissues (A). The statistical graph shows the densitometric analysis of TGF- $\beta 1$ (B), $\alpha-S M A$ (C), and P-Smad2/3 vs. Smad2/3 (D) expression normalized to $\beta$-actin expression. All experiments were repeated at least 3 times, and similar results were obtained each time. $\mathrm{N}=3$ (per group), data are presented as mean $\pm S D$, analyzed by one-way ANOVA followed by Bonferroni post hoc testing. ${ }^{*} \mathrm{P}<0.05$, vs. control group. $\# \mathrm{P}<0.05$ vs. adenine group. 


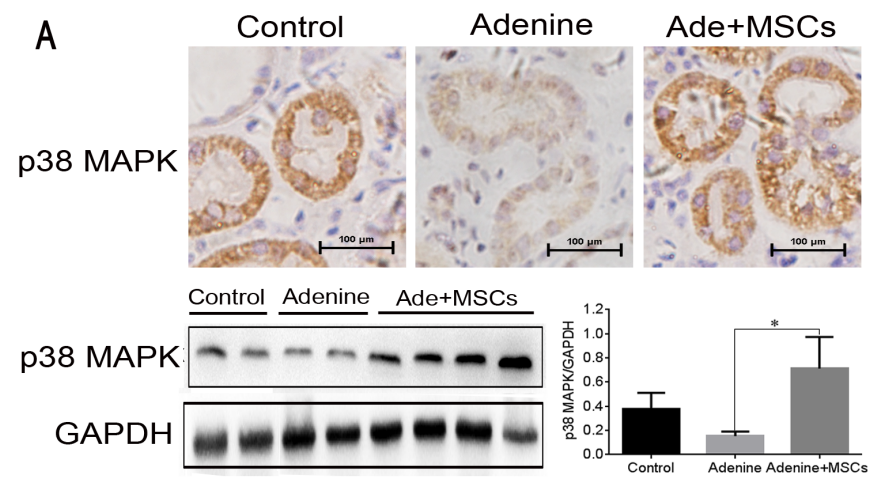

B

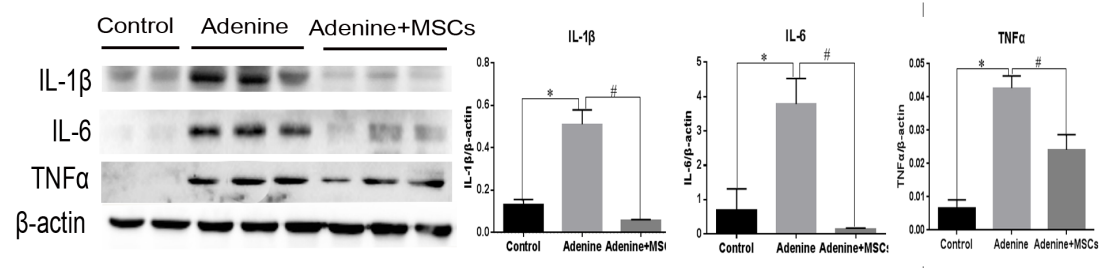

C

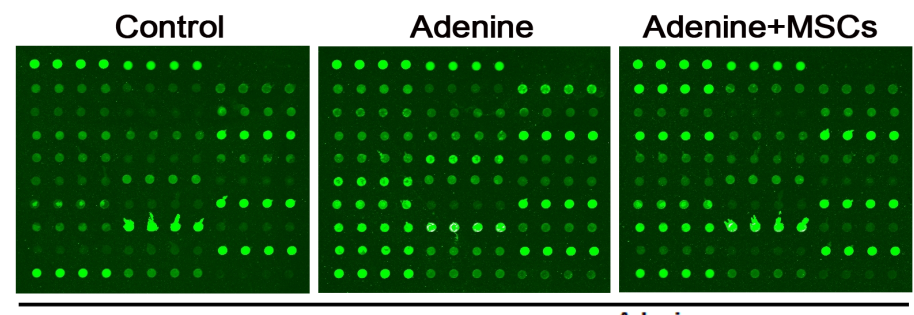

\begin{tabular}{lcccc}
\hline Name & Control_VS_Adenine & $\boldsymbol{P}$ Value & $\begin{array}{c}\text { Adenine } \\
\text { VS_Adenine+MSCs }\end{array}$ & $\boldsymbol{P}$ Value \\
\hline B7-2 & 0.93 & 0.79 & 0.52 & 0.30 \\
D-NGF & 1.22 & 0.36 & 0.64 & 0.08 \\
CINC-1 & 0.72 & 0.55 & 1.14 & 0.79 \\
CINC-2 & 0.59 & 0.19 & 1.30 & 0.44 \\
CINC-3 & 0.35 & 0.39 & 1.69 & 0.59 \\
CNTF & 1.28 & 0.55 & 0.67 & 0.18 \\
Fractalkine & 1.52 & 0.16 & 0.48 & 0.22 \\
GM-CSF & 0.85 & 0.34 & 1.31 & 0.12 \\
CAM-1 & 0.49 & 0.01 & 1.03 & 0.88 \\
FNg & 0.87 & 0.41 & 1.32 & 0.13 \\
L-1a & 0.23 & 0.19 & 1.92 & 0.41 \\
L-1b & 1.60 & $\leqslant 0.001$ & 0.87 & 0.23 \\
L-2 & 0.25 & 0.15 & 2.81 & 0.21 \\
L-4 & 1.24 & 0.11 & 0.77 & 0.11 \\
L-6 & 0.64 & 0.50 & 1.11 & 0.86 \\
L-10 & 0.36 & 0.02 & 1.49 & 0.28 \\
L-13 & 1.27 & 0.43 & 0.63 & 0.16 \\
LIX & 1.16 & 0.22 & 0.80 & 0.21 \\
L-Selectin & 0.43 & $\leqslant 0.001$ & 1.06 & 0.71 \\
MCP-1 & 0.82 & 0.09 & 1.04 & 0.61 \\
PDGF-AA & 0.61 & 0.30 & 1.30 & 0.53 \\
Prolactin R & 0.12 & 0.25 & 2.66 & 0.42 \\
RAGE & 0.29 & 0.34 & 2.51 & 0.42 \\
TCK-1 & 0.79 & 0.21 & 0.93 & 0.72 \\
TIMP-1 & 0.81 & 0.15 & 1.19 & 0.42 \\
TNFa & 1.24 & 0.11 & 1.08 & 0.53 \\
VEGF & 0.26 & 0.07 & 1.38 & 0.48 \\
\hline & & & &
\end{tabular}

\section{Figure 7}

MSCs activated p38 MAPK signaling and reduced inflammation in adenine-induced rats. A. Immunohistochemistry and western blot of p38 MAPK in kindey tissues. B. Western blot analysis of IL-6, IL-1 $\beta$, and TNFa in kidney tissue. Representative immunohistochemical staining images in kidney sections (scale bar, $100 \mu \mathrm{m}$ ) and representative western blot images of p38 MAPK protein at 5 days postMSCs treatment. The statistical graph shows the densitometric analysis of p38 MAPK expression 
normalized to GAPDH expression, and of IL-6, IL-1 $\beta$, and TNFa expression normalized to $\beta$-actin expression. All experiments were repeated at least 3 times, and similar results were obtained each time. $\mathrm{N}$ $=3$ (per group), data are presented as mean $\pm S D$, analyzed by one-way ANOVA followed by Bonferroni post hoc testing. ${ }^{*} P<0.05$, vs. control group. $\# P<0.05$ vs. adenine group. $C$. Microarray analysis of cytokine antibodies revealed that a total of 17 cytokines increased in the serum, such as CINC-1, CINC-2, CINC-3, GM-CSF, ICAM-1, IFNY, IL-1a, IL-2, IL-6, IL-10, L-Selectin, MCP-1, PDGF-AA, Prolactin R, RAGE, TCK1, TIMP-1, and VEGF, and a total of 8 cytokines decreased, including b-NGF, CNTF, Fractalkine, IL-1 $\beta$, IL-4, IL-13, LIX, and TNFa in adenine group. Among them, there were significantly statistical differences both increases in ICAM-1, IL-10, and L-Selectin and reduction in IL-1 3 . While MSCs treatment reduced the serum levels of 18 upregulated cytokines and increased the serum levels of 8 downregulated cytokines. $\mathrm{N}$ = 5 (in each group), the mean value of four replicates was first calculated as the signal value of each factor, then the signal value was normalized to positive control to allow comparison between subarrays, and finally the concentration was relatively quantified by using the normalized data. The intergroup ratio of 27 factors was calculated, and the P values between groups were analyzed by t-TEST (double-tailed). 
A

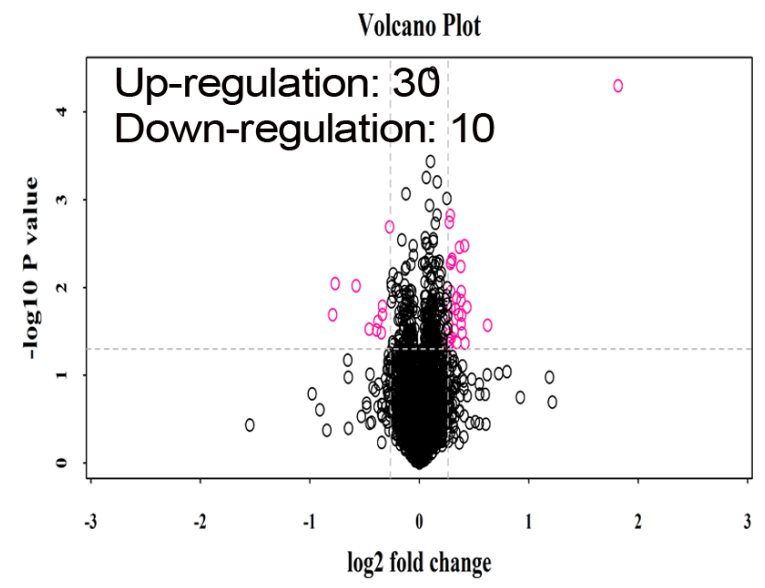

B

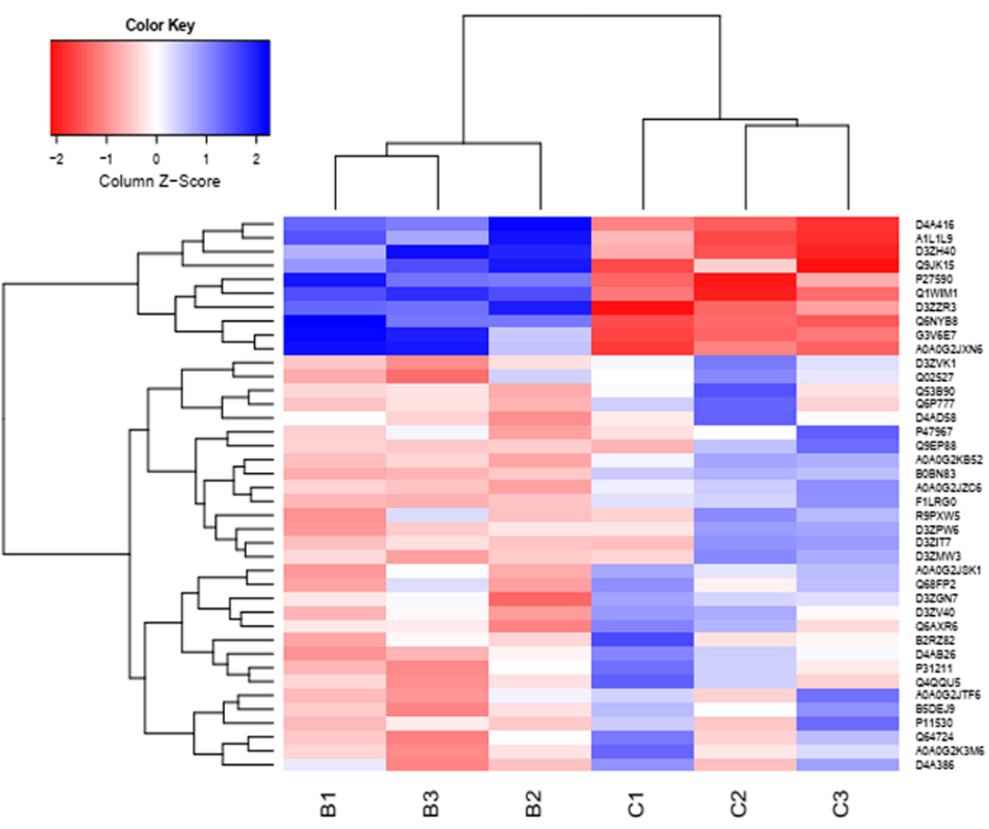

C

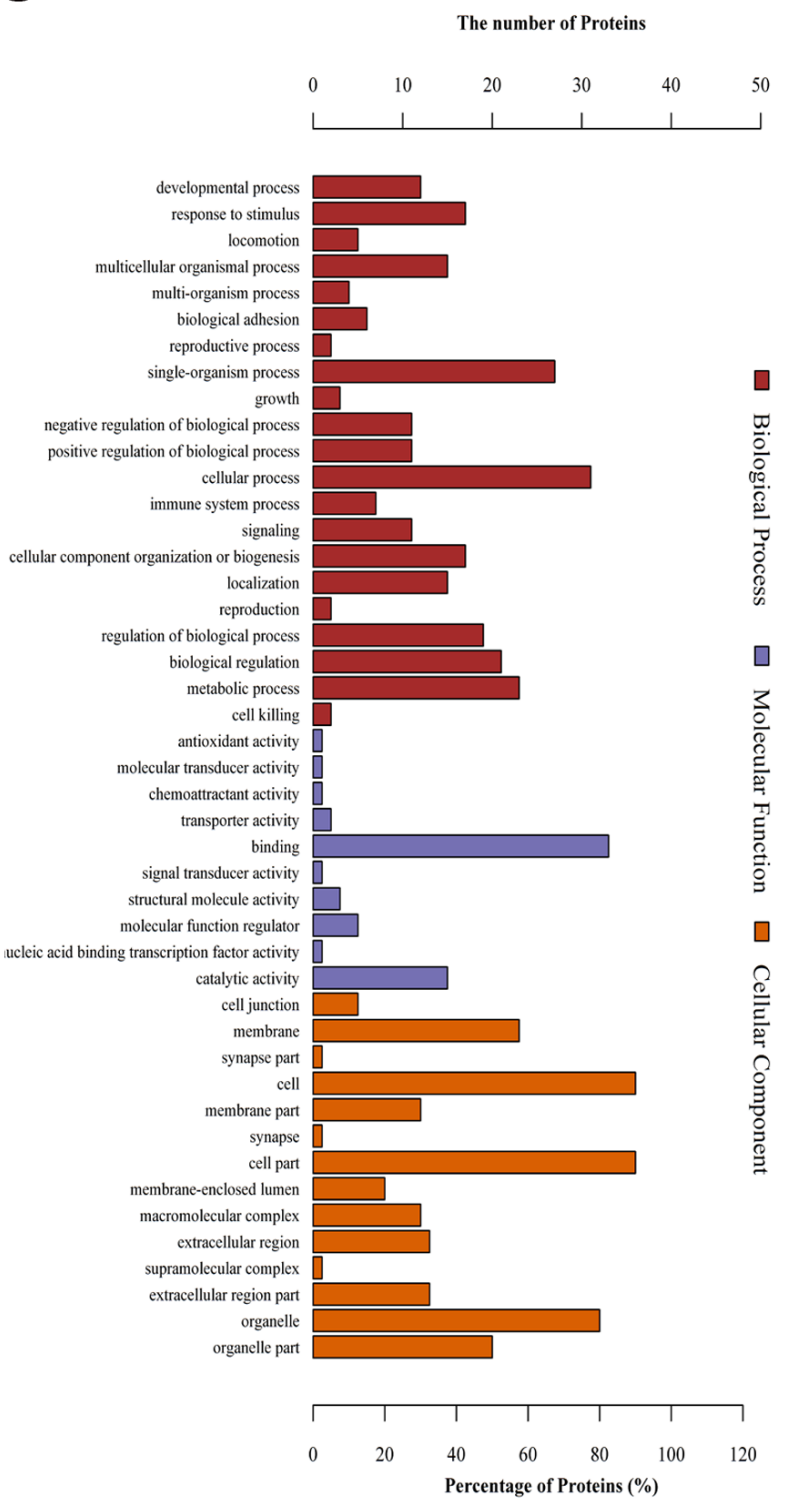

\section{Figure 8}

Global protein expression patterns in adenine-induced rat kidneys. (A) Volcano plots showing the distribution of significance and fold change of identified proteins in the Adenine+MSCs group/Adenine group comparison. All proteins were plotted with log2 (fold change) on the $x$-axis and log10 ( $p$-value) on the $y$-axis. Vertical dotted lines mark a fold change of $\pm 120 \%$ and the horizontal dotted line marks $P=$ 0.05. (B) K-means clustering representation of total 40 different expression proteins (DEPs). The magnitude of the percentage is represented by a color scale (top right) going from low (red) to high (blue). (C) GO annotation and functional classification of DEPs: Geneontology terms for subcellular location distribution, molecular functions, and biological process. 
A

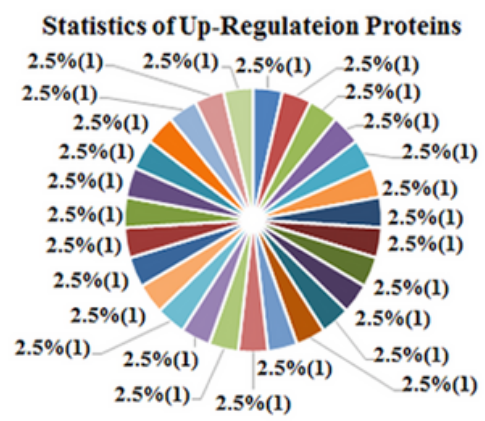

- Dilated cardiomyopathy

" N-Glycan biosynthesis

- Hepatitis C

- Leukocyte transendothelial migration

- MAPK signaling pathway - plant

- Bisphen ol degradation

- Metabolism of xenobiotics by cytochrome P450

- Glutathione metabolism

- Pathways in cancer

- Viral myocarditis

- Thiamine metabolism

- Hypertrophic cardiomyopathy (HCMI)

- Purine metabolism

- Chemical carcinogen esis
B

Statistics of Down-Regulateion Proteins

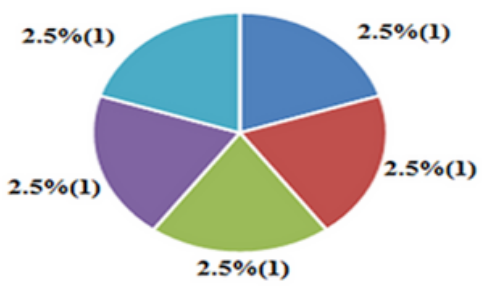

= Antigen processing and presentation = Tuberculosis

" Apoptosis

C
- Cell adh esion molecules (CAMs)

= Pyrimidine metabolism

= Arrhythmogenic right ventricular cardiomyopathy (ARVC)

- Vascular smooth muscle contraction

- Platinum drug resistance

- Aldoster one synthesis and secretion

- Fluid shear stress and atherosclerosis

- Endocytosis

= Tight junction

- Aminobenzoate degradation

- Drug metabolism - cytochrome P450

= Steroid hormone biosynthesis

- Signaling pathways regulating pluripotency of stem cells

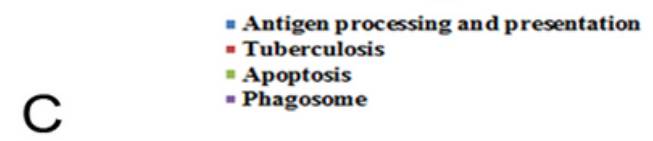

KEGG Pathway with significant statistically differences (Up-regulation proteins)

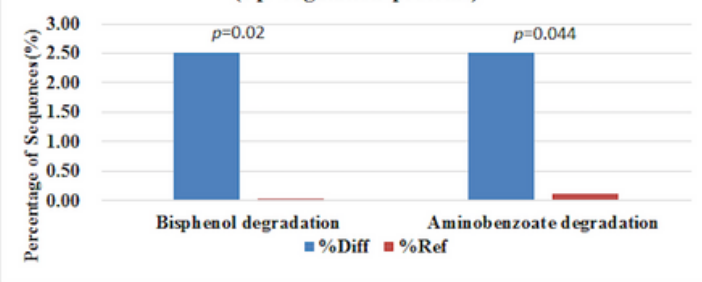

\section{Figure 9}

KEGG pathway analysis of the differentially expressed proteins compared Adenine group with Aadenine+MSCs group, including statistics of up-regulated proteins (A) and statistics of down-regulated proteins (B). Among these pathways of up-regulated proteins, bisphenol degradation and aminobenzoate degradation (C) were significantly statistical difference compared Adenine group with Adenine+MSCs group. p value was 0.02 and 0.04 , respectively.

\section{A}

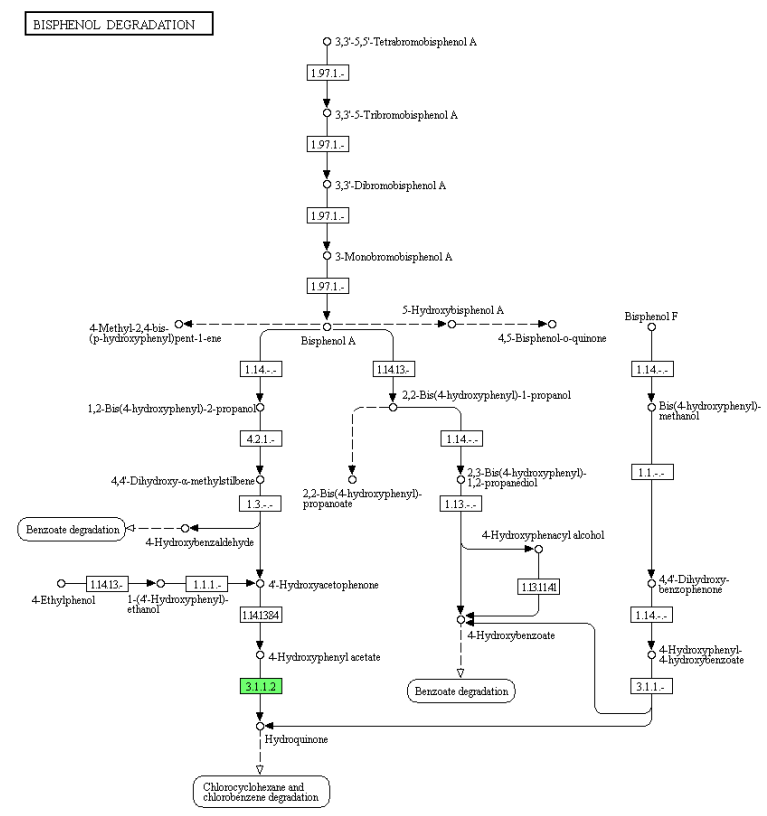

B

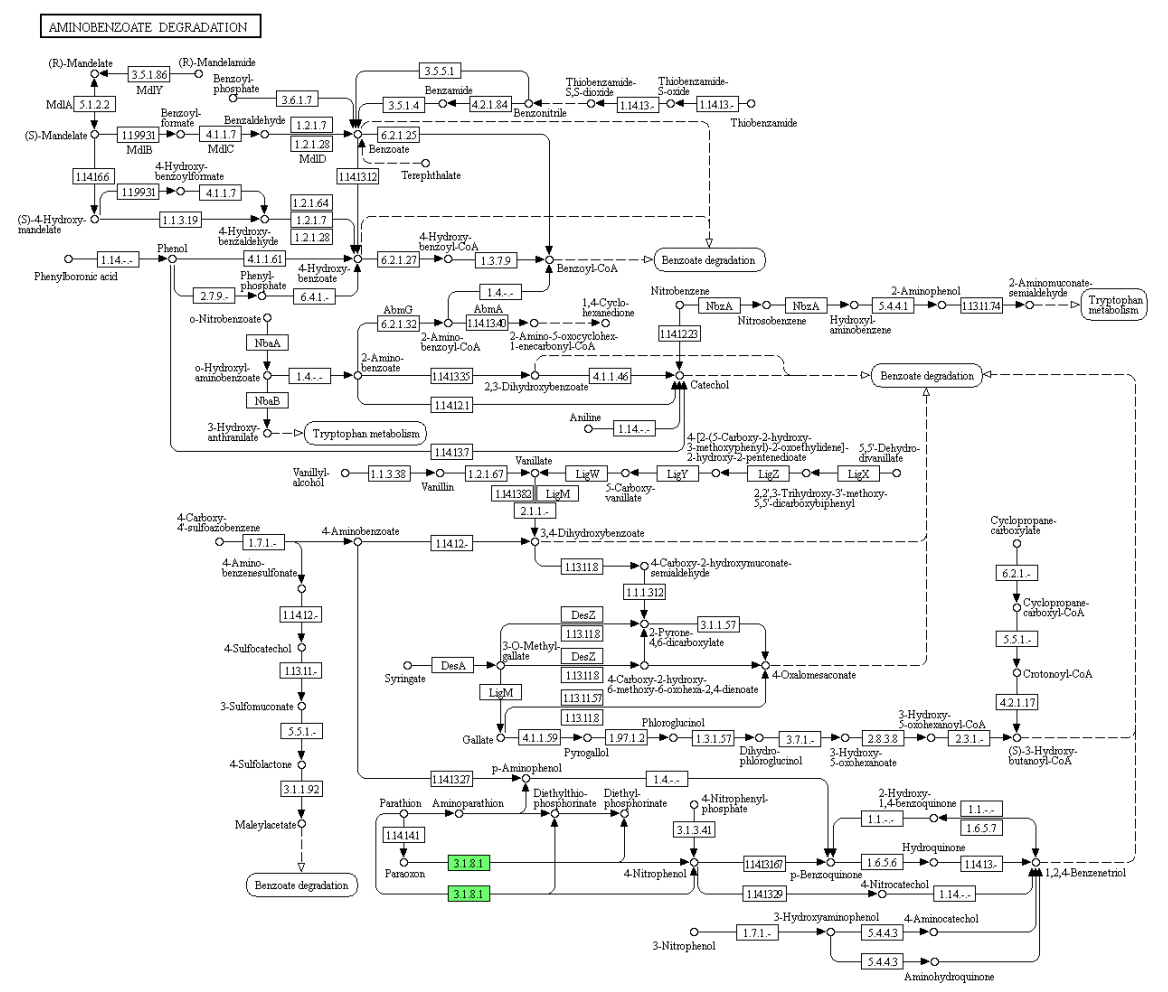


Bisphenol degradation diagram (A) and aminobenzoate degradation diagram (B) from KEGG pathway analysis for up-regulated proteins compared Adenine+MSCs group with Adenine group.

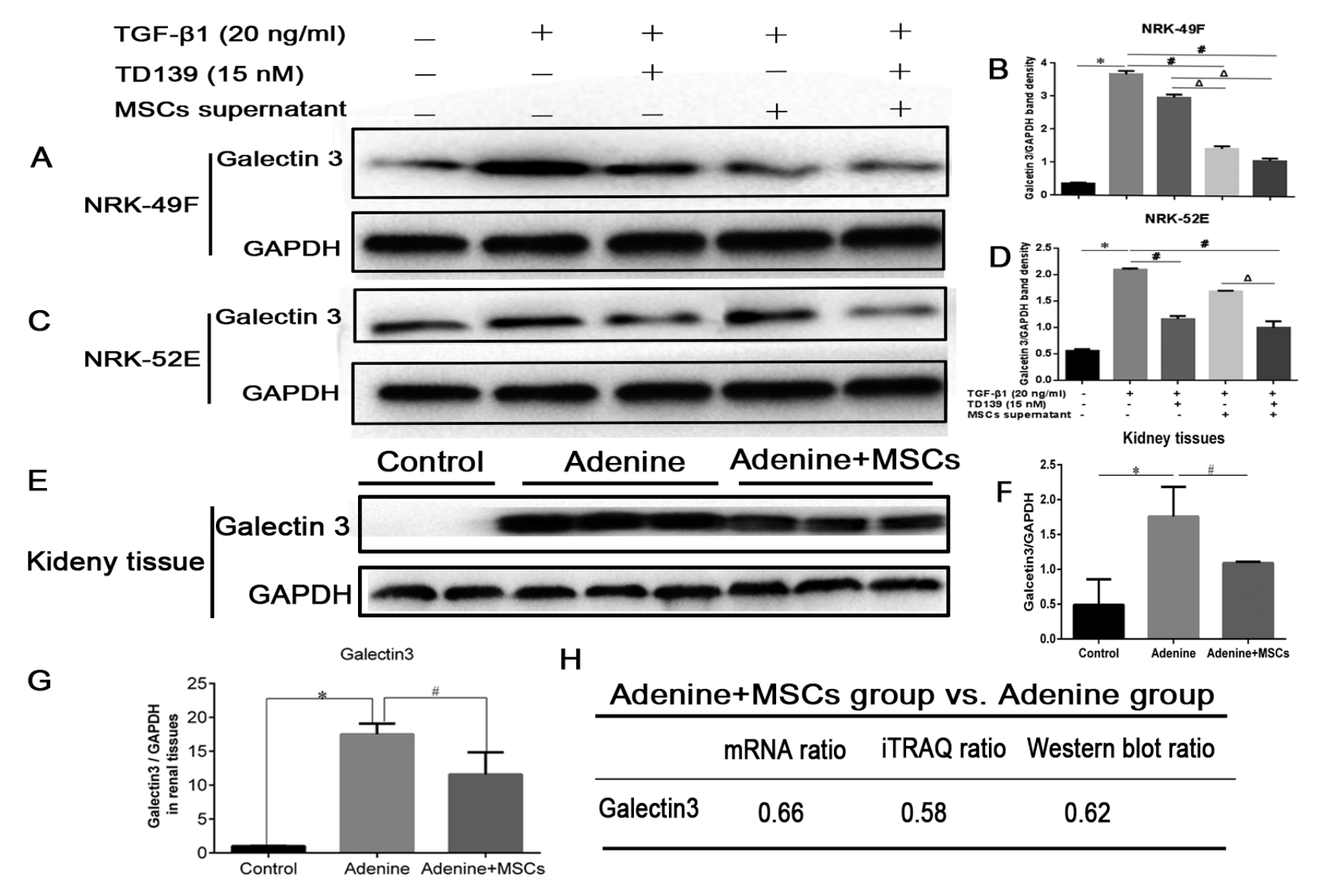

Figure 11

In vitro, confirmation of differentially expressed protein (galectin3) expression in MSCs conditioned medium treatment for NRK-49F (A, B) and NRK-52E (C, D) induced by human recombinant TGF- $\beta 1$ (20 $\mathrm{ng} / \mathrm{mL})$ with or without galectin3 inhibitor, TD139 $(15 \mathrm{nmol} / \mathrm{L})$ pretreatment by immunoblotting analysis. In vivo, confirmation of differentially expressed protein (galectin3) by western blot (E, F) and Q-PCR $(G)$ in adenine-induced kidney tissues post-MSCs treatment. Q-PCR ratios, WB ratios and ITRAQ ratios (Adenine/Adenine+MSCs) were shown on the Figure $11 \mathrm{H}$. The GAPDH protein was used as a control. Representative mRNA, representative immunoblotting and densitometric analysis of galectin3 expression in kidney tissues and cells. Results were normalized relative to the expression of GAPDH. $\mathrm{N}=3$ (per group), data are presented as mean $\pm S D$, analyzed by one-way ANOVA followed by Bonferroni post hoc testing. ${ }^{*}<<0.05$, vs. Control group, $\# P<0.05$, vs. Adenine group in kidney tissues; ${ }^{\text {, }} \# P<0.05$, vs. TGF- $\beta 1$ group, $\triangle P<0.05$, vs. TGF- $\beta 1+T D 139$ group in NRK-49F cells; ${ }^{*}$, $\# P<0.05$, vs. TGF- $\beta 1$ group, $\triangle P<0.05$, vs. TGF- $\beta 1+$ MSCs group in NRK-52E cells. Western blot analysis showed decreased levels of galectin3 post- 
MSCs treatment in the adenine-induced rats and cells, TD139 pretreatment further reduced galectin3 expression. 